\title{
A state-transition model of team conceptual design activity
}

\author{
Martinec, Tomislav; Škec, Stanko; Horvat, Nikola; Štorga, Mario
}

\section{Published in:}

Research in Engineering Design

Link to article, DOI:

10.1007/s00163-018-00305-1

Publication date:

2019

Document Version

Peer reviewed version

Link back to DTU Orbit

Citation (APA):

Martinec, T., Skec, S., Horvat, N., \& Štorga, M. (2019). A state-transition model of team conceptual design activity. Research in Engineering Design, 30(1), 103-132. https://doi.org/10.1007/s00163-018-00305-1

\section{General rights}

Copyright and moral rights for the publications made accessible in the public portal are retained by the authors and/or other copyright owners and it is a condition of accessing publications that users recognise and abide by the legal requirements associated with these rights.

- Users may download and print one copy of any publication from the public portal for the purpose of private study or research.

- You may not further distribute the material or use it for any profit-making activity or commercial gain

- You may freely distribute the URL identifying the publication in the public portal

If you believe that this document breaches copyright please contact us providing details, and we will remove access to the work immediately and investigate your claim 
This is a post-peer-review, pre-copyedit version of an article published in Research in Engineering Design. The final authenticated version is available online at: http://dx.doi.org/10.1007/s00163-018-00305-1

\title{
A state-transition model of team conceptual design activity
}

\author{
Authors: \\ Tomislav Martinec ${ }^{\mathrm{a}} \quad$ (ORCID: 0000-0002-6487-4749) \\ Stanko Škec a,b $\quad$ (ORCID: 0000-0001-7549-8972) \\ Nikola Horvat ${ }^{\mathrm{a}} \quad$ (ORCID: 0000-0002-6354-2734) \\ Mario Štorga ${ }^{\mathrm{a}, \mathrm{c}} \quad$ (ORCID: 0000-0001-9700-008X)
}

a Department of Design, Faculty of Mechanical Engineering and Naval Architecture, University of Zagreb Ivana Lučića 5, 10000 Zagreb, Croatia

b Technical University of Denmark, DTU Management Engineering

Produktionstorvet, Building 424, 2800 Kgs. Lyngby, Denmark

${ }^{\mathrm{c}}$ Division of Humans and Technology - Department of Business Administration, Technology and Social Sciences, Luleå University of Technology

97187 Luleå, Sweden

\section{Corresponding author:}

Tomislav Martinec

tomislav.martinec@fss.hr

\begin{abstract}
:
The purpose of the study is to model the micro-scale process patterns which can be identified during team conceptual design activities. A state-transition model has been developed and used to empirically investigate the patterns of design operations during two types of team conceptual design activities: ideation and concept review. The presented work builds on the perception of design problems as ill-defined and implies that conceptual design activities involve simultaneous development of problems and solutions using three distinctive design operations analysis, synthesis and evaluation. The three design operations have been defined as fine-grain design steps performed by design teams when exploring the content of both the problem and the solution dimensions of the design space. Moreover, design operations have been conceptualised as transitions between states of the explored design space, thus providing a basis for the state-transition model. The model's ability to map and visualise proportions of design operation sequences emerging during ideation and concept review has facilitated identification of both the activity-specific patterns and patterns that were likely to appear during both types of empirically investigated activities. The two activities exhibited similar patterns, such as alternation of solution synthesis and analysis, sequences of synthesis, analysis and evaluation within solution space, and the potential coevolution episodes. Nevertheless, divergent traits have been identified for ideation, and convergent traits for concept review, based on the significant differences in proportions of design operations and their sequences.
\end{abstract}

\section{Keywords:}

Design process, Conceptual design activity, Teamwork, State-transition model, Ideation, Concept review 


\section{Introduction}

The conceptual design phase has a significant impact on the final design outcome as it transforms the initial formulation of a design problem into a clear description of a concept solution, by reducing the unknown and assuring that the subsequent phases are mainly technical (Kroll 2013; Andreasen et al. 2015). Due to the nature of design problems, a large part of the unknown lies within the design problem itself. The conceptual design phase thus assumes reciprocating decomposition of design problems and exploration of possible solutions before a final concept is proposed (Liikkanen and Perttula 2009). This simultaneous evolution of design problems and solutions is believed to be the driver of creativity in the early development activities, especially in team designing (Wiltschnig et al. 2013). Hence, not only does the majority of engineering designers in modern industrial practice work as part of a team, but the creative conceptual design tasks such as idea-generation or concept selection are often performed exclusively as team activities (Sonalkar et al. 2013; Toh and Miller 2016a).

Even though team conceptual design activities are potentially the most creative, vibrant and dynamic from the designers' point of view, they are also the least understood by the researchers (Macmillan et al. 2001). For example, the insufficient understanding of conceptual design activities has been reflected in lack of adequate computeraided support in the conceptual design phase and human-computer interfaces which do not consider the nature of the designing (Vuletic et al. 2018). Proper models of the actual design processes have thus become essential for understanding designers and developing tools that could assist them in designing (Goldschmidt, 2014), specifically, design teams in formulating design problems and providing solutions to these problems throughout the conceptual design phase.

Many prescriptive models of design make use of the perspective of design as a problem-solving process (Lawson and Dorst, 2009), where designing should start with a clarification of the design problem, followed by generation and evaluation of the solution alternatives before a single solution is selected. However, Cross (2001) explains that in the case of ill-defined problems, designers do not typically start by pursuing to define the design problem rigorously. They instead progressively and iteratively discover, structure and address the issues as they emerge in the design process (Wynn and Eckert 2017). Even when designers follow a systematic problem-solving strategy, they continuously generate new task goals and redefine task constraints (Visser 2009). Two distinctive dimensions of design space - the problem space and the solution space - are developed through a constant iteration of analysis, synthesis and evaluation (ASE) processes (Dorst and Cross 2001). The above portrayal of designing by Dorst and Cross has been embraced across a number of studies in design research (see, e.g. Wiltschnig et al. 2013, Liu and Lu 2014, Eckert et al. 2012, Jin and Chusilp 2006, Khaidzir and Lawson 2013, Afacan and Demirkan 2011). Moreover, as shown throughout the paper, both the notion of problem-solution space and ASE as fundamental processes have regularly been employed in investigation and modelling of design activity.

Nevertheless, while the proposal of problem-solution co-evolution in design research (Maher et al. 1996) has been around for over twenty years, the questions of how exactly ASE sequences iterate and intertwine throughout the conceptual design phase, and in what way these patterns differ for the problem and the solution space, have not been extensively explored. Fine-grain approaches, used to understand the details of micro-scale cycles (Wynn and Clarkson 2017) in conceptual design activities, have either employed ASE sequences within the solution space (e.g. Stempfle and Badke-Schaub 2002, Gero et al. 2013), neglected the evolution of both spaces (e.g. Fiorineschi et al. 2016), or focused solely on individual designers (e.g. Mc Neill et al. 1998; Sarkar and Chakrabarti 2014). Additionally, empirical studies have generally been tied to only a specific type of conceptual design activity, such as ideation (e.g. Gero et al. 2013; Cardoso et al. 2016) or concept review and selection (e.g. Toh and Miller 2015, 2016a,b). The utilisation of diversified team compositions, coding schemes and modelling approaches in these studies hinders direct comparison of the results. Because of the inability of a proper inter-study comparison and due to the lack of studies offering a simultaneous investigation of team designing across different activities, such as ideation and concept review, there exists little insight on how the micro-scale process patterns are affected by the design activity goal and team's progress within the conceptual design phase.

The presented research aims to fill the gap in modelling the micro-scale conceptual design process of teams performing analysis, synthesis and evaluation in both the problem and the solution space and understanding how the design processes differ for ideation and concept review activities. Insights into patterns of ASE and their effect on the evolution of the explored design space should complement the existing models of team design activity and increase the understanding of team conceptual design process. The following research questions have guided the study:

- What patterns of analysis, synthesis and evaluation altering inside and in-between the problem space and the solution space can be identified during team conceptual design activities?

- In what way do the identified patterns differ for two types of team conceptual design activity, namely the ideation and the concept review?

The study is divided into two main parts: theoretical and empirical. The first, theoretical part focuses on the review of relevant literature and the development of a model of team conceptual design activity. In the empirical part, the experiments with teams conducting two types of conceptual design activities are analysed based on the developed model in order to address the research questions. 


\section{Background}

Design researchers have adopted numerous perspectives of the engineering design process to study the activity of designing in teams. Depending on the phenomena of interest, studies have investigated design through the lens of thinking (Stempfle and Badke-Schaub 2002), creativity (Toh and Miller 2016a), information processing (Cash and Štorga 2015), problem-solving (Kurakawa 2004), co-evolutionary design progression (Wiltschnig et al. 2013), learning (Deken et al. 2012), decision-making (Brissaud et al. 2003), and more.

While there exist differences in the way researchers explore and model design, its multifaceted nature is well recognised (Lawson and Dorst, 2009; Cash and Štorga, 2015). For instance, in their domain-independent descriptive model of design, Reymen et al. (2006) introduce the notion of design situation, which combines three facets of design: the state of the product being designed, the state of the design process, and the state of the design context. According to their model, designing is the activity of transforming the state of the product being designed or of the design process into another state towards the design goal. They also use the concept of design space to refer to possible states of the product and the process. The state of the design context, on the other side, is separated from designing and is changed by the stakeholders (e.g. user requirements, company norms, available production technologies, etc.). Moreover, while designing is affected by the context it takes place in, the context itself most often does not change within the time span of design activities (Reymen et al. 2006), such as ideation sessions. Hence, according to design situation viewpoint, team design activities represent sequences of designers' actions towards a design goal, which result in the evolution of the explored design space (transforming the state of the product and the process) considering the specific (static) design context.

Jin and Benami (2010) introduce the notion of design operations when referring to the observable, fine-grain acts of designing. In their generate-stimulate-produce (GSP) model, design operations are used to generate design entities, which in return stimulate designer's thinking processes, leading to new design operations. The model gives a clear distinction between the observable aspects of the designing such as talking, writing and sketching, and the internal ones such as the underlying thinking processes of designers (Jin and Benami 2010). GSP was initially utilised to investigate creative patterns and stimulation of individual designers but was later expanded into collaborative thought stimulation (CTS), where design entities are shared by team members (Sauder and Jin 2016). By adopting the introduced notions, design operations are from here on conceptualised as the observable, finegrain acts performed by design teams to transform states of the design space. However, since the CTS model focuses on design operations as generators of design entities, the notion of design operation must be adjusted to reflect all of the fundamental ASE processes, performed in both the problem and the solution dimension of the design space. Before the proposed conceptualisation of team design activity is further elaborated, several areas of relevant research on both individual designers and design teams are examined. As a starting point, the empirical studies of team design thinking are considered, to provide a better understanding of what drives design operations in design teams. Next, the role of ASE and design space evolution across different models of the design process is investigated. Finally, some insights on the ideation and concept review activities are briefly discussed. The examined areas are, however, not mutually exclusive. For example, the empirical studies often utilise observable design operations and the change in design space as a proxy for investigating the thinking processes of designers.

\subsection{Empirical studies of team design thinking}

Given the viewpoint of design thinking as an underlying process of designing, particular attention has been given to modelling of designers' thinking processes. For many years now, design researchers have been employing approaches such as think-aloud and conversational methods, case studies and controlled experiments to explore the thinking patterns during execution of design tasks (Dinar et al. 2015). Design thinking research is inspired by other disciplines currently studying collective thought, including social and cognitive psychology, organisational sciences and anthropology (Stompff et al. 2016). Fine-grain investigations of the designing have thus often been carried out using protocol analysis, currently the most suitable method of revealing the cognitive abilities of designers (Cross 2001). Reported protocol studies of design teams are mainly concurrent and conversational (Hay et al. 2017), meaning that the participants concurrently report on their thinking acts using conversation during task execution. The resulting cognitive models usually describe the iterative nature of designing in which design alternatives are repeatedly generated, analysed and evaluated through exploration and convergence (Wynn and Clarkson, 2005; Wynn and Eckert, 2017).

A noteworthy example is the generic model of design team activity by Stempfle and Badke-Schaub (2002), who employed protocol analysis to capture regularities in thinking and reasoning processes underlying the problemsolving process of three laboratory teams. Their study proposes a process that matches the "natural" thinking process of design teams, where the generation of solution ideas is followed by immediate evaluation, except when there are any questions or misunderstandings. If such quick evaluation yields a positive result, teams decide to accept the solution. Otherwise, new solution ideas are sought (Stempfle and Badke-Schaub 2002). Ensici et al. (2013) provided additional detail to the decision process by focusing on the phenomena of using and rejecting decisions, based on whether they have been included in the final solution. They decomposed the team design process into thinking processes related to decision making and identified the consequences of rejected decisions, such as narrowing the solution space and prioritisation, structuring and complexity reduction of the design problem 
(Ensici et al. 2013). In their analysis of collaborative sketching, Eris et al. (2014) discuss the significant role of gestures in team designing. For example, they identify that gestures which construct conceptual relations between two sketches (cross-gestures) facilitate the shared understanding of designers. Sauder and Jin (2016) focused on generative thinking processes of memory retrieval (when an experience or design entity that existed in the past is remembered), association (when connections are drawn between two design entities), and transformation (when a design entity is altered or changed). They observed that the stimulation occurring through questioning has the strongest relationship with the generative thinking processes. Cardoso et al. (2016) investigated design thinking in teams during ideation as an inquiry-driven process. They observed patterns of cognitive moves triggered by reflection on dissatisfaction and facilitated by formulation of high-level questions that steer the direction of the design discourse. Sung and Kelley (2018) analysed sequences of cognitive strategies of design teams and identified a bi-directional iteration between designing and predicting, or simply put - introducing ideas and predicting possible consequences of the ideas.

Although the above-listed studies provide valuable insights into team design thinking, the used protocol coding schemes are closely tied to the specific context and the phenomena observed, making it difficult to directly compare the results and conclude how team design activity is affected by the change in design context or progress of the design process.

In contrast to the use of different coding schemes, a portion of empirical research on design team thinking processes utilised the function-behaviour-structure (FBS) ontology of design and designing. These studies have accepted the axiom that "the foundations of designing are independent of the designer, their situation and what is being designed" (Gero and Kannengiesser 2014). Kan et al. (2011) utilised the FBS ontology to observe an industry team brainstorming session and measure frequencies of transitions between FBS design issues and interactions on the individual and team level. Jiang et al. (2014) applied the same ontological framework to study design cognition of small teams within the context of different disciplines and conceptual design tasks. They classified the teams' designing styles as problem- and solution-focused. As an extension of that research, Gero and Jiang (2016) studied the design review and critique sessions. Both studies reveal commonalities across designing but also identify the differences between design domains and design tasks. Gero et al. (2013) investigated how different creativity techniques reflect in design cognition of team members during the concept generation activity. They coded the activity of eleven design teams and found a correlation between the structuredness of ideation techniques and design teams' focus on the problem or solution-related aspects of designing.

In the case of employing the unified FBS coding scheme, different episodes in the design process can be investigated and compared, particularly the cognitive processes regarding the design space (functions, behaviours and structures). However, FBS being an ontology that primarily describes the design as an artefact, the elements of the design process are derived from transitions between the coded segments, rather than being directly coded. Additionally, since only transitions between certain pairs of FBS design issues are assigned with micro-scale processes, the FBS coding scheme may not be suitable for addressing the study's research questions.

Within the context of the presented research, the abovementioned empirical studies of team design thinking are relevant for two main reasons. Firstly, they provide valuable methodological insights into the development of a protocol analysis study. Secondly, the studies have contributed an extensive collection of insights into different aspects of team behaviour, which can be utilised for comparison, interpretation, validation and discussion of the protocol study results.

\subsection{Analysis, synthesis and evaluation as design operations}

All design processes are different unless examined at a very abstract level (Eckert and Clarkson 2005). Studies aimed at unfolding the commonalities amongst designing in different domains confirm this by indicating that only at the high level of abstraction can similarities between different domains be recognised (e.g. Reymen et al. 2006; Kannengiesser and Gero 2015). Any comparison of different individuals, teams, activities, domains or methods, whether in search for similarities, patterns or differences, stems from the prerequisite of abstraction in modelling both the design process and the design space. Therefore, the descriptions of individual or small team design activities have predominantly been given in the form of abstract micro-scale models, which emphasise the iterative nature of designing and the need of responding to new information generated or revealed during the design process (Wynn and Clarkson 2017). A well-adopted example of such abstraction implies the sequences of analysis, synthesis and evaluation (ASE). These three fundamental processes (operations) can be traced back to Asimow (1962), who proposed ASE model as a general problem-solving strategy, and Watts (1966), who presented the design process as iterative cycling through ASE.

Initially, the ASE sequence was intended as a model of sequential phases in the design process but was often criticised for not reflecting the reality of design projects (Visser 2009). The more or less defined sequences of ASE were later incorporated within micro-scale models of design. Abstract processes analogous to ASE can thus be identified across eminent descriptive models of design. For example, the "basic design cycle" by Roozenburg and Eekels (1995) consists of analysis, synthesis, simulation, evaluation and decision. The "design steps" by Gero (1990), which represent the transitions within the FBS ontology, include analysis, synthesis, evaluation, formulation and reformulations. ASE is included in the "iterative processes" between the problem and the solution space in creative design by Dorst and Cross (2001). The "generic step model" of design team activities by Stempfle 
and Badke-Schaub (2002) consists of generation, analysis, evaluation and decision. The "integrated model of designing" (IMoD) by Srinivasan and Chakrabarti (2010a) classifies generic activities into generate, evaluate (which can also include analysis), modify and select. Abstraction using ASE can also be found within models of problem-solving in design (Aurisicchio et al. 2013) and creative process models (Howard et al. 2008). To provide clarity, the term design operations by Jin and Benami (2010) is adopted when referring to ASE as the observable fine-grain steps within the design process (as opposed to the phases in the design process).

The insufficiently understood role of ASE design operations in the co-evolution of the problem and solution space is in part a result of inconsistency in the interpretation of ASE as fine-grain steps in the design process. Firstly, depending on their purpose, the models of design tend to associate analysis to either the problem or the solution space. The prescriptive design models inherit the problem-solving interpretation of ASE, where analysis is a step performed within the problem space and includes understanding, decomposition and formulation of design requirements (Wodehouse and Ion 2010; Fiorineschi et al. 2016). Although such instantiation of analysis can also be found in some of the descriptive approaches (e.g. Roozenburg and Eekels 1995; Mc Neil et al. 1998), the others of the aforementioned descriptive models associate analysis to solution space, where it is performed to increase the understanding of solutions prior to evaluation. These models introduce concepts such as formulation (Gero 1990), goal clarification (Stempfle and Badke-Schaub 2002) and problem definition (Casakin and Badke-Schaub 2017) to summarise the design operations in the problem space. Secondly, although synthesis has been shown to play an equally important role in developing both design problems and solutions (Smithers 2002), its integration as part of the ASE sequence within prescriptive and descriptive models is primarily in the form of a solution generation (McTeague et al. 2017). Thirdly, with new entities populating the problem space as designing proceeds, the co-evolutionary process implies not only the need for evaluation of design solutions but also evaluation of the introduced requirements and constraints (Maher and Tang 2003; Hay et al. 2017). However, the term evaluation has mainly been used to describe the assessment of design solutions concerning the problem being solved, e.g. in the FBS framework (Jiang et al. 2014), problem-solving steps of design teams (Stempfle and Badke-Schaub 2002) or the creative processes in design (Howard et al. 2008). Problem evaluation remains a phenomenon that has not been explicitly included within the reviewed ASE design models.

Given the perspective of the simultaneous evolution of design problems and solutions (Dorst and Cross 2001), and ASE being regarded as different modes of conceptual thinking (Wodehouse and Ion 2010), the context of presented research calls for adopting the definitions of ASE as design operations performed in both the problem and the solution space. Rather than attributing each design operation to the problem or the solution space, the definitions should highlight the differences between analysis, synthesis and evaluation as fundamental mechanisms for evolving the design content.

\subsection{Co-evolution of the problem and the solution space}

In general, the design process is not linear whereby design problems could initially be fully defined and then solutions directly derived from them (Brissaud et al. 2003). The nonlinearity is particularly evident in the conceptual design phase, where designers iteratively perform ASE to drive the parallel evolution of problem and solution spaces (Dorst and Cross 2001; Eisenbart et al. 2017). The notion of problem-solution co-evolution has been introduced within the co-evolutionary model of designing by Maher et al. (1996) and has been present in many studies ever since, especially within the creative design research. In the model, designers are iteratively developing concepts and exploring the two spaces, with each space informing the other. Maher and Tang (2003) later investigated co-evolutionary design as a cognitive and a computational model of design and demonstrated the similarity of reasoning between the human designer's cognition and computational algorithms co-evolutionary cycles. However, studies have also shown that despite the commonalities in types of cognitive strategies employed by human designers, their focus on problem or solution space can differ and that the co-evolution strategies can be distinguished as problem- and solution-driven (Kruger and Cross 2006). In their domain-independent descriptive model of design, Reymen et al. (2006) have implemented the problem-solution co-evolution as a simultaneous evolution of desired and current properties. Hultén et al. (2018) have related their model of ideation to problem-solution co-evolution by introducing the concepts of common ground and transformative closure. The first implies returning to the problem space with a new understanding of the problem, and the latter implies reaching a solution space that can develop and change during the process. They emphasize the need for conceptualizing the common understanding (ground) as support for co-evolution within the models of designing in teams (Hultén et al. 2018).

The generality and applicability of co-evolutionary design are yet to be comprehensively tested for team-based conceptual design. Recent studies support the co-evolution during collaborative activities such as ideation (Cash and Storga 2015) and concept selection (Toh and Miller 2015), but also throughout a series of real-world product design meetings (Wiltschnig et al. 2013). Moreover, a study by Deken et al. (2012) has shown an increased alternation between the spaces during conceptual design compared to the task clarification phase.

The explored design space (problem and solution) evolves as new design entities are generated, and the existing ones are modified. Different types of design entities appearing in the problem and the solution space have been abstracted in more-less similar ways. For example, the reasoning about requirements, functions and expected behaviour of the artefacts within the FBS ontology is related to the problem space, and reasoning about structure 
and behaviour as observed of the structure is related to the solution space (Jiang et al. 2014). Macmillan et al. (2001) have used the terminology of needs, requirements and problems as conceptual design entities in problem space, and solutions, proposals and concepts as entities in solution space. In their study of solution- and problemdriven design, Kruger and Cross (2006) categorised the problem entities into requirements and constraints. Sarkar and Chakrabarti (2014) recognised requirements, related problems, constraints, solutions and evaluation criteria. Liikkanen and Perttula (2009) used the terms goals and subgoals in the exploration of problem decomposition. On the other hand, the IMoD by Srinivasan and Chakrabarti 2010a classifies entities of the problem-solution space solely into generic requirements and solutions, thus eliminating the issue of vague boundaries between some of the terms describing entities in the design space. For example, functions and behaviour (see Dorst and Vermaas 2005; Eisenbart et al. 2017), requirements and constraints (Ball et al. 2010), or how ideas become concept solutions (e.g. Howard et al. 2010; Srinivasan and Chakrabarti 2010b; Sonalkar et al. 2013). Based on these findings, here presented research will not consider detail classification of design entities but will instead use the concepts of problem and solution space to cover the full range of design entity expressions.

\subsection{Protocol studies of ideation and concept review}

Ideation (idea generation) and concept review are considered core activities within the overall design process, due to their creative potential and impact on the final design outcomes (Cash and Štorga 2015; Toh and Miller 2015). Significant research efforts have thus been directed towards prescribing approaches, methods and tools to facilitate generation of high-quality ideas and selection of the best concept solutions in a particular context. The prescriptive research has primarily been aimed at boosting creativity and productivity, but also overcoming fixation and bias (please consult Gonçalves et al. 2014; Vasconcelos and Crilly 2016; Petersson and Lundberg 2018; Nikander et al. 2014; and Toh and Miller 2016b for more details on recent findings concerning these issues). Although there are many formalised methods developed for ideation and concept selection, it is often suggested that designers prefer working informally rather than using the less intuitive and imposed methods (Toh et al. 2014; Nikander et al. 2014). Understanding the designers' natural and intuitive approach to designing (both the cognitive and the observable process) is essential in providing teams better support during activities such as ideation and concept review.

Despite the acknowledged need for understanding the naturally occurring process of designing, fine-grain decomposition of ideation and concept review processes has rarely been in focus of design research. Moreover, the comparison of the two activities is, again, hindered by the use of different coding schemes, team formations, design environments, etc. Nevertheless, some additional process and behaviour patterns identified across the protocol studies of ideation and concept review are discussed below.

Sarkar and Chakrabarti (2014) studied idea generation of individual designers and identified different patterns of design space search taking place during problem understanding, solution generation, and solution evaluation, and related the types of searches to solution quality. They later propose a model of ideation where, given an unsolved problem, designers find an existing, related solution from the past (from memory), and then they modify it for the current problem, whether in the phase of problem formulation, solution generation or solution evaluation (Sarkar and Chakrabarti 2017). Liikkanen and Perttula (2010) also perceive ideation as a memory-based activity which consists of memory sampling and idea production. They pointed out that individual designers generate similar initial ideas before contextual cueing and verbal stimulation are introduced. However, a semantically substantial and associatively rich change of context and verbal stimulation are shown to alter the ideation process.

Stimulation has also been studied on team-level. López-Mesa et al. (2011) studied the effect of stimuli coupled with individuals' problem-solving styles on the ideation process of design teams. They argue that stimulus with images leads to a higher quantity of solutions, while stimulus by idea-prompting checklist favours refinement of solutions. Sauder and Jin (2016) employed retrospective protocol analysis and found that collaborative prompting and clarification have a strong relationship with remembering design entities, while collaborative seeding and correcting strongly correlate to altering and changing design entities.

Cash and Štorga (2015) have explored what drives innovation by using network analysis to link ideation to the engineering context and the broader design process. Insight derived from the networks include identification of decoupled ideation, characterised by producing numerous solution ideas, and integrated/iterative ideation, expressed in co-evolution of design problems and solutions. Hatcher et al. (2018) have embodied Linkography to compare the creative processes when two different ideation methods are used (brainstorming and an approach proposed by the authors). Their findings include, for example, that brainstorming has a less structured approach and is more likely to contain a higher number of idea moves (idea generation) inspired by non-idea moves, such as questions (idea analysis).

Protocol studies of concept review (and the more general design review) activity have primarily been in focus of design research with educational implications (see, e.g. Adams et al. 2016), such as guidelines for mentors who provide feedback, advice or critique. However, little is known about the team-based concept review process and how teams select creative ideas (Toh and Miller 2016a). Moreover, while concept review is generally described as a convergent activity (Nikander et al. 2014), Toh and Miller (2015) note that team members often not only evaluate and select concepts, but also combine, modify, and propose new solution ideas. They point out that teams who pursue to generate new ideas during concept review tend to select more creative concepts. 
The FBS framework has also been used to investigate different types of design activities. As mentioned earlier, it was employed to study design cognition during both ideation (e.g. Kan et al. 2011; Gero et al. 2013; Jiang et al. 2014) and concept review (Gero and Jiang 2016). Since the FBS ontology offers commensurability of study results (Gero and Kannengiesser 2014), these studies can be qualitatively compared. Gero and Jiang (2016) identified the similarity between the two activities in the linearity of cumulative occurrences of structure and behaviour issues, however, they noticed that unlike designing (ideation), concept review activity does not exhibit the decrease in the ratio of the problem- and the solution-related discussion as the session progresses. Aside from these efforts, the micro-scale descriptions of how teams synthesise, analyse and evaluate design entities during concept review remain undeveloped.

Within the context of the presented research, the studies of ideation and concept/design review offer insight into key characteristics of these activities, thus complementing the general findings resulting from empirical research on team design thinking when discussing the protocol analysis results.

\section{Framing the team conceptual design activity}

Considering the diversity in interpretation of ASE within the reviewed micro-scale models of design, the first step in framing the team conceptual design activity implies adapting clear definitions of analysis, synthesis and evaluation. The definitions should reflect the conceptualisation of ASE design operations as fundamental mechanisms for evolving/co-evolving the design entities within both problem and solution space. Furthermore, the team conceptual design activity has been constrained within the domain-independent descriptive model of design by Reymen et al. (2006). In the model, the evolution of the explored design space (problem and solution) is represented by a set of states, while designing is the activity of transforming one state to another. If designing is decomposed into design operations, then ASE design operations express the transitions between the states of the design space. Fig. 1 illustrates the sequences of design operations as transitions driving the evolution of the explored design space (change of the state of the product being designed and the state of the design process) while approaching the goal of the design activity.

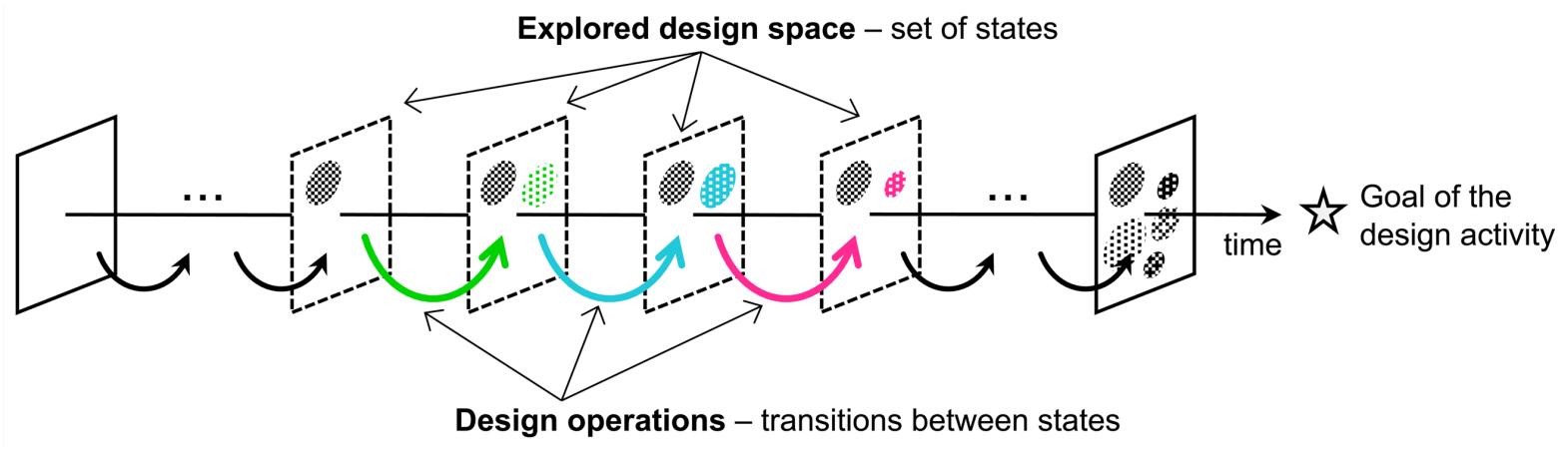

Fig. 1 Design operations as transitions between the states of the explored design space (adopted from Reymen et al. 2006)

In the presented research, the ASE design operations have been defined by adapting the categorisation system for verbal activities in design teams by Casakin and Badke-Schaub (2017). This categorisation presumes exploration of both the problem and the solution dimensions of the design space. Hereafter, the ASE design operations as transitions between the states of the explored design space have been defined as follows:

- Analysis is a state transition resulting in an increased understanding of a particular design entity within the explored design space. When performed in problem space, the purpose of the analysis is to clarify the design problem (needs, requirements, constraints, etc.). The purpose of performing analysis in solution space is to increase the understanding of the proposed solutions to the problem (ideas, concepts, alternatives, etc.). Problem analysis corresponds to "clarification of aspects and questions related to design issues, i.e. user, technical, or budget issues", while solution analysis corresponds to "analysis of a solution idea or part of it", as defined by Casakin and Badke-Schaub (2017).

- Synthesis is a state transition resulting in the appearance of a new design entity within the explored design space. Solution synthesis corresponds to "stating a new idea or a new solution for a problem or subproblem developing new aspects of an earlier solution idea" (Casakin and Badke-Schaub 2017). Improvement, refinement and combining of solution entities are also considered as synthesis design operations since the original design entities remain in the solution space, and new derivatives appear. Problem synthesis corresponds to "definitions that are considered in order to structure and define the problem" (Casakin and Badke-Schaub 2017).

- Evaluation is a state transition resulting with the assessed utility of a particular design entity within the explored design space. Evaluation of a design entity (in problem or solution space) is performed by 
addressing a criterion, that is the relevant design entity in the problem space (requirement, constraint, etc.). Two different scenarios of performing evaluation have been identified based on the problem decomposition techniques described by Liikkanen and Perttula (2009). In the first one, the problem space design entities (criteria) are explicitly identified before the execution of evaluation design operation. In the second scenario, the problem entities (criteria) are introduced implicitly within the team at the moment of performing evaluation design operation. Although the goal in both cases is assessing the suitability of a particular design entity (problem or solution), in the second scenario, a new problem entity (criterion) emerges in parallel to the evaluation design operation. McDonnell (1997) described the scenario of detecting "misfits" during solution evaluation, which can lead to reframing the problem. In a similar matter, Harvey and Kou (2013) explain that the role of evaluation during creative group tasks is not only to provide feedback and make decisions but also to frame the problem. Solution evaluation corresponds to "assessment of a solution idea by focusing on its value and feasibility" as defined by Casakin and Badke-Schaub 2017. They, however, do not propose any verbal activities concerning problem evaluation. Nevertheless, the proposed framework assumes that design entities appearing in the problem space can likewise be evaluated. Hence, problem evaluation is considered as a means of assessing the utility of the new requirements, constraints or subgoals.

The fundamental difference between synthesis and analysis is that as a result of the synthesis design operation a new design entity appears in the explored design space. The fundamental distinction of evaluation design operations is that it also envelops the criterion by which the manipulated design entity is assessed. Fig. 2 illustrates how ASE design operations act as transitions between the states of the explored design space. The illustration has been simplified by merging the problem and the solution dimensions of the design space. It is important to notice that Fig 2. illustrates only a single scenario of performing a sequence of ASE design operations and that it does not imply that such sequence is dominant in designing.

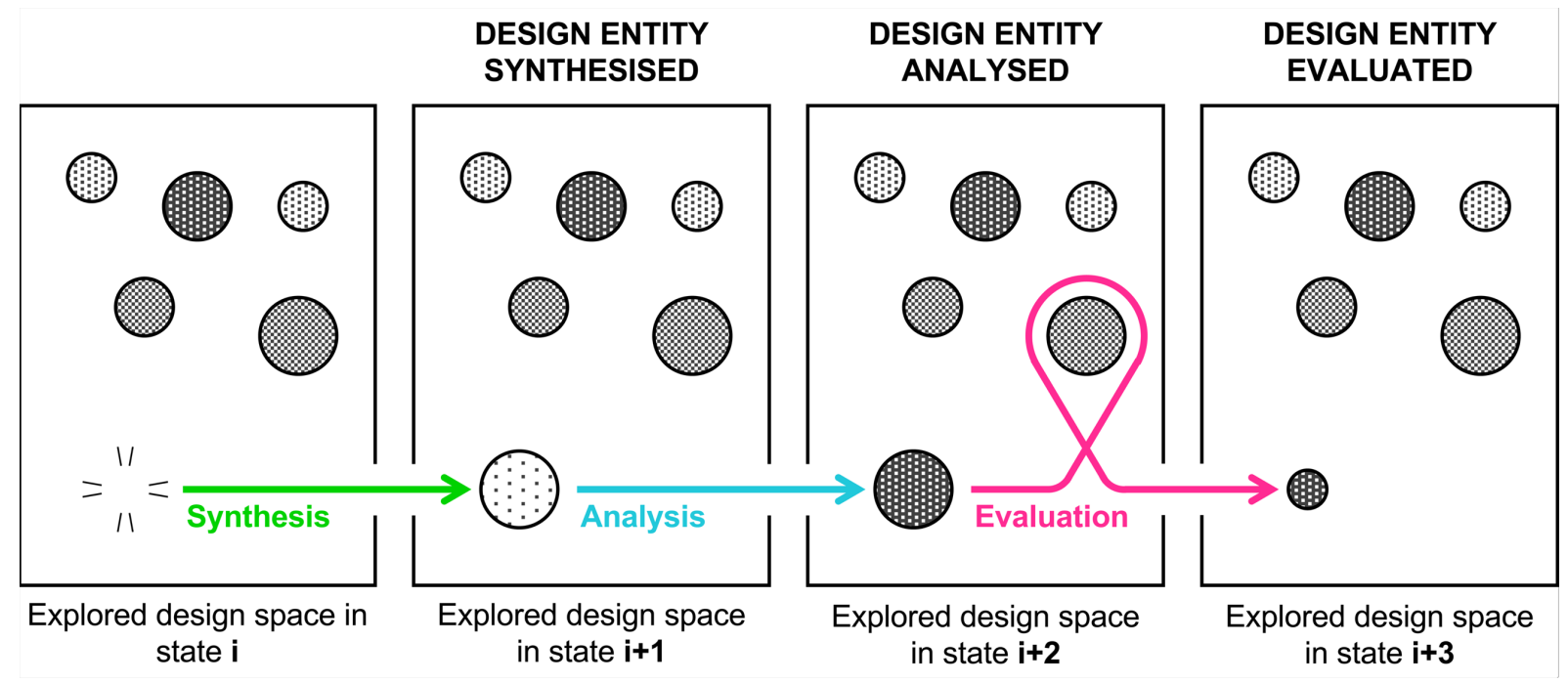

Fig. 2 Illustration of a state-transition sequence

In state $\mathrm{i}$, the explored design space is likely to be populated with both problem and solution entities. If members of the design team perform, for example, a synthesis design operation, a new design entity (problem or solution) is revealed (as a result of the transition from state $i$ to state $i+1$ in Fig. 2). The new design entity can be either entirely unrelated to existing ones (new or global searches according to Sarkar and Chakrabarti 2014) or elaboration and improvement of the existing design entities (local and detail searches).

If team members perform analysis design operation, they increase the individual or shared understanding of a design entity (transition from state $i+1$ to state $i+2$ in Fig. 2). The aim of analysis can be to avoid misunderstanding (Stempfle and Badke-Schaub 2002) or to improve the understanding of certain aspects of design entities (e.g. to determine the behaviour of a solution, as seen by Gero 1990). The better the design entity is understood, the bolder it appears in the Fig. 2 state illustrations.

Finally, as designers progress through conceptual design activity, they require convergent action to narrow down the choices (Yilmaz and Daly 2016; Goldschmidt 2016). Designers thus evaluate problems and solutions to distinguish the ones that are reasonable and acceptable. When team members perform evaluation design operation, they asses the utility of a design entity concerning the relevant criteria (the assessed utility of the design entity changes as a result of a transition from state $i+2$ to state $i+3$ in Fig. 2). The size of the design entities in Fig. 2 illustrates the assessed utility. 


\subsection{State-transition model of team conceptual design activity}

The proposed definitions of ASE design operations fit within the framework presented in Fig. 1 by matching the transitions between the states of the design space. In addition, a micro-scale design process model has been added to the framework to capture the dynamics of these transitions during a team conceptual design activity. According to McMahon (2015), fine-grain design process elements such as design operations can be modelled as state transitions. Therefore, the dynamics of the micro-scale design process of team conceptual design activities are here described using a state-transition model. The model's visualisation is shown in Fig. 3.

The state nodes in the model represent the states of the design space after ASE design operations have been performed. Once the design entity has been analysed, the state within the model changes to "design entity analysed", as shown in Fig. 2 and 3. Similarly, the synthesis design operation changes the state to "design entity synthesised" and evaluation to "design entity evaluated".

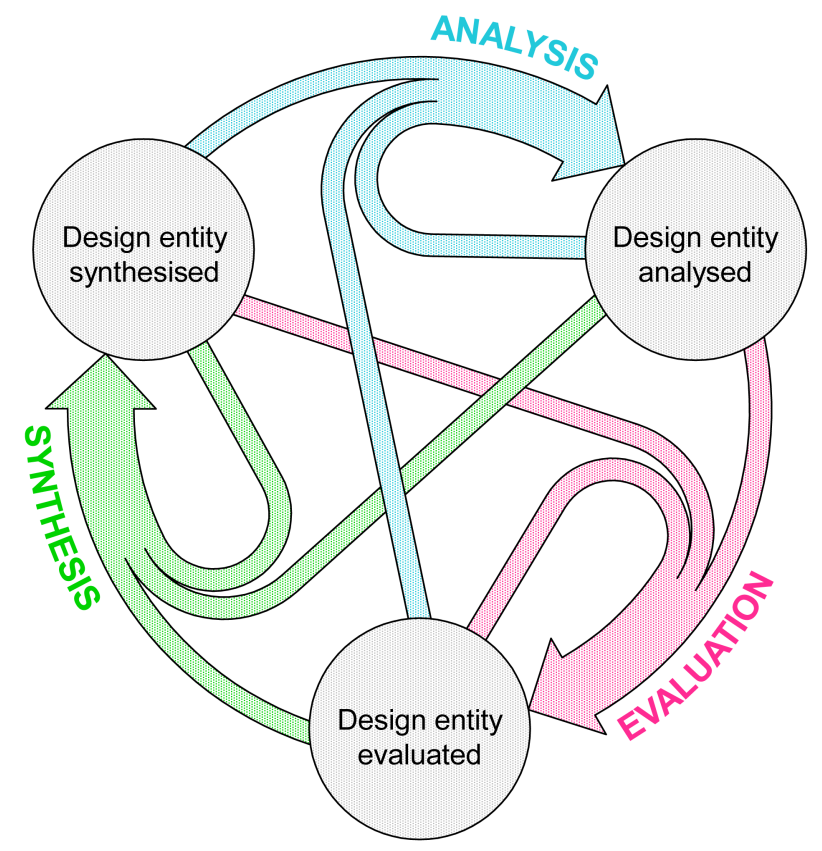

Fig. 3 State-transition model visualisation illustrating ASE design operations performed within the design space

All transitions between the state nodes can be assigned with a probability of being performed by the team. These probabilities can be expressed as constant throughout the activity (based on average probabilities of transitions during the whole activity), or they can be formulated as a function of time passed since the start of the activity, or the number of entities present in the design space. For example, once team members have synthesised a design entity, they have made a change to the design space. The micro-scale design process is now in the "design entity synthesised" state and the team can carry out further design operations. They can perform analysis, synthesis or evaluation, each with a certain probability assigned within the current state node. If the next step is a synthesis of a new design entity, the transition will return into the same node which will then represent the next state of "design entity synthesised" (now with one additional entity). If the team, however, performs analysis or evaluation, the state node changes along the corresponding transitions. The change in the state node also results with new probabilities for ASE transitions in the following step (e.g. the most probable transition after analysis might be a synthesis, but once synthesis is carried out the most probable transition might then be evaluation).

Rather than having a sequential nature, the model's flexibility allows iterative cycles of one or two types of design operations. For example, the model can reflect the sequences of ASE design operations driven by divergent and convergent thinking (Yilmaz and Daly 2016; Goldschmidt 2016), where a single or a pair of design operations dominate. Such descriptions are important since new design entities do not appear at a constant pace, nor is every new design entity analysed and evaluated (Liu et al. 2003).

The model visualised in Fig. 3 considers design space as one-dimensional (without dividing it to problem and solution space) for the simplicity of representation and clarification. As such, the model consists of three states and nine transitions between these states. Nevertheless, the model can easily be extended to map also ASE transitions within and in-between the problem and the solution space, thus providing additional insight into the co-evolution of these two dimensions. In this way, each transition is divided into four subtypes: two within the spaces (solution to solution and problem to problem), and two in-between the spaces (problem to solution and solution to problem). With these four subtypes of transitions, the model gets more complex as the number of possible transitions rises to 36 . 
The visualization of ASE transitions in both spaces is presented in Fig. 4. Additional colour codes have been added to highlight transitions within and in-between the problem and the solution space. Transitions within the spaces reflect the evolution of a single space (problem or solution) and transitions in-between the spaces reflect how teams switch from one space to another and thus drive the co-evolution of problems and solutions.

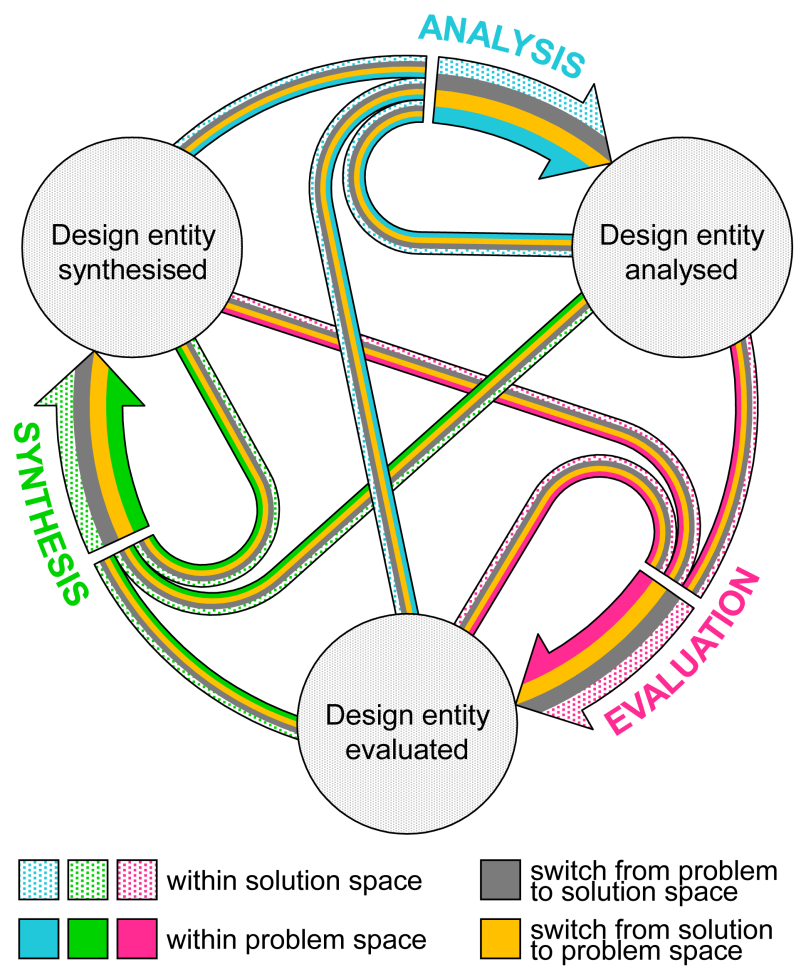

Fig. 4 State-transition model visualisation illustrating ASE design operations performed within and in-between the problem and the solution space

The visualisation of the state-transition model can be further enhanced by assigning thickness to the state transition edges (arrows) based on the proportion of ASE design operations during the team conceptual design activity. In this manner, the relative thickness of a single transition in comparison to other transitions corresponds to the ratio of the matching design operation and all possible design operations during the activity. Empirically based examples of visualising the transition proportions are presented in the results section.

\section{Empirical study}

Guided by the studies investigating fine-grain patterns in team design activity, the empirical part of the presented research has been conducted in the form of a verbal protocol analysis study. There are several reasons for selecting verbal protocol analysis as the principal means of investigating team conceptual design activity. Firstly, conceptual design communication in design teams is primarily verbal (Frankenberger and Auer 1997; Andreasen et al. 2015). Secondly, the concern regarding the validity of verbalisations in teamwork is irrelevant, since it is natural for team members to verbally communicate when working together, making verbal data an authentic reflection of real-time thinking in design teams (Goldschmidt 2014). Thirdly, since the presented research focuses on the observable design operations, the segments when designing is not (or cannot be) verbalised are not documented and modelled as observable steps of the design process.

In the light of research questions set out and the research methodology applied, the aim of utilising protocol analysis was the identification of fine-grain patterns of ASE design operations during team ideation and concept review activities. Methodologically, the study consists of four main stages: (1) development of a theoretical framework, (2) obtaining and describing the experimental data set, (3) segmentation and coding, and (4) data analysis and interpretation of the results.

The theoretical development has been presented in previous sections. The second step was focused on gathering of empirical data, namely defining criteria for selecting the appropriate recordings of team conceptual design sessions. In the third step, the recordings of conceptual design sessions were segmented and coded. The coding scheme for verbal protocol analysis was defined accordingly to the theoretical framework of team conceptual design activity established in step one. Lastly, in the final step, the protocol data were analysed and discussed. 


\subsection{Experiment session participants and tasks}

Several features were considered when identifying the appropriate empirical data set. The recorded experiment sessions should have been collaborative activities, where teams engage in tasks of conceptual design nature. The duration of the task execution should have been relatively short (e.g. no more than two hours) due to the use of protocol analysis method, but long enough to collect a sufficient number of data points. Furthermore, teams should have participated in two different types of activities within the conceptual design phase, to address the second research question.

Video recordings of the two types of team conceptual design activities were obtained from previously conducted studies by Cash et al. (2013). The decision to use existing raw recording data provided several benefits. First, the received data set meets the study requirements, so conducting new experiments could have been avoided. Second, the data set results from a rigorously designed experiment and has already passed several cycles of thorough examination, peer review and publication. Third, studies that used the same raw data set provide additional insights into the design process and offer the potential of coupling the results.

The original experiment structure consisted of four sessions, two of which were team activities - ideation and concept review. The other two activities of the experiment were performed by designers individually and are thus not in focus of here presented research. Nevertheless, to provide context for the team sessions, the complete experiment structure is introduced. In each experiment session, the participants were given a task - a formulation of a design problem to be solved, aiming at the observation and the examination of the design process (Bender 2003). The participants were first engaged in individual information seeking task, particularly for feasibility level technical information on camera mounting devices. This individual task was followed by a collaborative ideation activity, in which participants were grouped into teams of three and given a design brief to deliver concept ideas for mounting a camera on a balloon. After the team sessions, participants have again worked on individual design tasks to develop a single, elaborated concept. Finally, the teams met again to review and refine the concepts (Cash et al. 2013). The practice of divergent ideation, followed by elaboration and integration of ideas, and completed by narrowing and refining ideas is not unique to design, as similar progress can be found across creative group task processes (Harvey and Kou 2013).

A total of twelve participants were randomly allocated to four teams. The teams were composed of mechanical engineering students selected from a final year product design and development course. Each participant had an average of 10 months of industrial experience and four years of academic training background at the time of the experiment. For more information on the teams, please consult Cash et al. (2013) and Cash and Maier (2016).

\subsubsection{Team conceptual design activity 1 : Ideation}

During the ideation activity, the teams had 50 minutes to generate as many viable ideas as possible for a cameramounting concept hanged under a helium balloon. The concept should have been capable of mounting any camera and orienting it to any point in a hemispherical region. The solution must have been operated remotely. Teams could have recorded their ideas on the whiteboard and sheets of paper. The protocol was based on the participants' natural conversational acts (without imposing any verbalisation requirements), to reduce the effects of observation. For more information please consult Cash et al. (2013).

\subsubsection{Team conceptual design activity 2 : Concept review}

Team compositions for the concept review activity were the same as for the ideation activity. During the concept review activity, the teams had 50 minutes to review concepts they developed and elaborated during the individual concept generation task. They were instructed to select and develop one, or a combination of concepts and refine them into a final concept solution. More information can be found in Cash et al. (2013).

\subsection{Segmentation and coding}

As part of the verbal protocol analysis, the recorded team conversations have been transcribed and parsed into coded segments, which were then treated as units of analysis (Maher and Tang 2003; Goldschmidt 2016). The transcription process helped to familiarise with the data and to develop and refine the instructions for coding prior to performing the final segmentation and coding step. Moreover, two additional codes have been recognised and added to the coding scheme during the transcription process. The coding scheme used in the study is shown in Table 1.

The core of the scheme consists of six codes that match the adopted definitions of ASE design operations in the problem space (problem analysis, problem synthesis, problem evaluation) and the solution space (solution analysis, solution synthesis, solution evaluation). The remaining two codes have been added to capture communicative acts which are not related to the design space. The "process" code is used for discussions concerning the action plan within the session. Detailed process-specific codes were not considered as only a small amount of process-related discussion has been identified. Clear instructions about the design task have been given before sessions start, and there was no need for teams to realign the process often. All remaining communicative acts such as any kind of off-topic discussion, naming unrelated facts and joking were coded as "Other". An example of a segmented and coded transcript can be seen in Table 2 . 
Table 1 Coding scheme for annotating the segments of design team conversation

\section{Code}

Description
Coders'

reliability $\left(\kappa_{\mathrm{a}}\right)$

O: other above (urelated facts, joking, off-topic discussion)

The developed coding scheme was applied to the transcripts of the experiment sessions. The segments were coded following the "one-segment-with-one-code" principle (see Jiang et al. 2014). Each segment was assigned with only one of the eight codes based on the coder's critical judgment of recognising ASE design operations as defined in the previous section. Although the situations in which more than one designer was talking were rare, these segments were coded based on the statement that was more dominant and to which the discussion continued.

The protocols were coded by the primary researcher and a trained coder who was not involved in the development of the framework. The first (primary) coder coded the entire 50 minutes of the eight experiment sessions. The second (reliability) coder coded random 10 minutes intervals (20\% of total session duration) of each experiment session, in order to satisfy the proportion needed for the calculation of inter-rater reliability, as suggested by Klonek et al. (2016). Similar approaches to reliability analysis in research of design teams can be seen in the studies of Deken et al. (2012), Wiltschnig et al. (2013), Eris et al. (2014) and Snider et al. (2016).

Table 2 An excerpt of segmenting and coding the experiment transcripts

\begin{tabular}{|c|c|c|c|}
\hline Time & Participant & Segment transcript & Code \\
\hline $7: 34$ & $\mathrm{P} 1$ & $\begin{array}{l}\text { [weight restriction concerning the cameras is discussed] } \\
\text { So, we are saying from } 200 \text { grams to } 3 \text { kilograms... }\end{array}$ & PS (new constraint) \\
\hline $7: 49$ & $\mathrm{P} 3$ & You then might not be able to attach the full-frame camera... & PE (constraint evaluated) \\
\hline $7: 53$ & $\mathrm{P} 2$ & $\begin{array}{l}\text { I think it will be more reasonable to restrict the weight it } \\
\text { would lift to a range of decent cameras. }\end{array}$ & PS (constraint modified) \\
\hline 8:04 & $\mathrm{P} 1$ & $\begin{array}{l}\text { What about the attachment of the camera? } \\
\text { [requirement introduced earlier in the session] }\end{array}$ & PA (requirement analysed) \\
\hline $8: 10$ & $\mathrm{P} 1$ & $\begin{array}{l}\text { The standard camera attachment is this quarter inch } \\
\text { threaded screw... }\end{array}$ & SS (solution proposed) \\
\hline $8: 20$ & $\mathrm{P} 2$ & Screw...? The tripod mount? & \multirow{2}{*}{ SA (solution clarified) } \\
\hline $8: 22$ & $\mathrm{P} 1$ & Yes. & \\
\hline $8: 23$ & $\mathrm{P} 2$ & $\begin{array}{l}\text { Yeah, that's the thing. Everything has that one... From little } \\
\text { compacts right to the big DSLRs }\end{array}$ & \multirow{2}{*}{ SE (solution evaluated) } \\
\hline $8: 35$ & $\mathrm{P} 1$ & $\begin{array}{l}\text { [writing the solution proposal on the whiteboard] } \\
\text { So that is very standard... }\end{array}$ & \\
\hline
\end{tabular}


Since the presented verbal protocol analysis aims to investigate distribution and sequences of design operations, the outputs of the coding are depicted as strings of codes, which are defined by Quera et al. (2007) as event sequences. During the coding process, the coders need to identify the events of analysis, synthesis and evaluation within the problem and the solution space. For this reason, the strings of codes produced by two independent coders may differ in length, that is, the number and the alignment of coded segments are likely to be different. As a consequence, the calculation of Cohen's kappa - the typical approach to assessing the inter-rater reliability cannot be performed, as it is not clear how the two event sequences can be aligned. An approach by Quera et al. (2007) was utilised to calculate the event-based interpretation of kappa: the event alignment kappa $\left(\kappa_{a}\right)$.

GSEQ software (see, e.g. Klonek et al. 2016) was used to compute the event alignment kappa for each code. Both the overall event alignment kappa value $\left(\kappa_{a}=0.71\right)$ and the event alignment kappa values for particular codes (reported in Table 1) indicate substantial agreement between the two coders. Once the inter-rater reliability was assessed, all of the identified conflicts have been resolved, and the final event sequences were agreed.

\section{Results}

The results are presented in two parts. The first part reveals the frequencies of segments assigned with the different codes during the two experimental sessions, with a particular focus on the segments where teams performed design operations. In the second part, the transitions between the coded segments are analysed to identify the sequences of design operations. The ideation and concept review experiment sessions are first examined separately and are then compared to determine the significant differences. From here on, the experiment sessions will be referred to as ideation and concept review activities.

On average, 333 codes have been coded per team during the ideation activity, and 313 per team during the concept review activity. The discussion related to the problem and the solution space accounts on average for 293 segments per team during the ideation (85-90\% of all segments), and for 280 segments per team during the concept review activity $(87-92 \%)$. The process-related conversation has averaged at $6 \%$ during ideation, and at $5 \%$ during concept review, and other communicative acts between $5 \%$ and $6 \%$. The absolute frequencies of each coded segments and their aggregation to ASE design operations and problem-solution spaces during both activities are available in Table 3.

Table 3 Absolute frequencies of coded segments during ideation and concept review activities

\begin{tabular}{|c|c|c|c|c|c|c|c|c|c|c|c|c|}
\hline \multirow{2}{*}{ Code } & \multicolumn{6}{|c|}{ Ideation } & \multicolumn{6}{|c|}{ Concept review } \\
\hline & T1 & T2 & T3 & T4 & Mean & SD & T1 & T2 & T3 & T4 & Mean & SD \\
\hline Problem analysis (PA) & 51 & 33 & 44 & 30 & 39.50 & 9.7 & 21 & 18 & 14 & 16 & 17.25 & 3.0 \\
\hline Problem synthesis (PS) & 74 & 47 & 41 & 34 & 49.00 & 17.5 & 9 & 10 & 14 & 10 & 10.75 & 2.2 \\
\hline Problem evaluation (PE) & 23 & 13 & 26 & 13 & 18.75 & 6.8 & 3 & 5 & 8 & 3 & 4.75 & 2.4 \\
\hline Solution analysis (SA) & 32 & 70 & 66 & 46 & 53.50 & 17.8 & 128 & 90 & 132 & 75 & 106.25 & 28.1 \\
\hline Solution synthesis (SS) & 72 & 97 & 105 & 99 & 93.25 & 14.6 & 72 & 71 & 89 & 64 & 74.00 & 10.6 \\
\hline Solution evaluation (SE) & 15 & 47 & 59 & 36 & 39.25 & 18.7 & 81 & 56 & 77 & 53 & 66.75 & 14.3 \\
\hline Process (PROC) & 25 & 14 & 24 & 16 & 19.75 & 5.6 & 16 & 18 & 15 & 13 & 15.50 & 2.1 \\
\hline Other $(\mathrm{O})$ & 22 & 24 & 18 & 14 & 19.50 & 4.4 & 13 & 20 & 26 & 11 & 17.50 & 6.9 \\
\hline Total of all coded segments & 314 & 345 & 383 & 288 & 332.50 & 40.9 & 343 & 288 & 375 & 245 & 312.75 & 57.7 \\
\hline Analysis (PA+SA) & 83 & 103 & 110 & 76 & 93.00 & 16.1 & 149 & 108 & 146 & 91 & 123.50 & 28.6 \\
\hline Synthesis (PS+SS) & 146 & 144 & 146 & 133 & 142.25 & 6.2 & 81 & 81 & 103 & 74 & 84.75 & 12.6 \\
\hline Evaluation (PE+SE) & 38 & 60 & 85 & 49 & 58.00 & 20.1 & 84 & 61 & 85 & 56 & 71.50 & 15.2 \\
\hline Problem space $(\mathrm{PA}+\mathrm{PS}+\mathrm{PE})$ & 148 & 93 & 111 & 77 & 107.25 & 30.5 & 33 & 33 & 36 & 29 & 32.75 & 2.9 \\
\hline Solution space $(\mathrm{SA}+\mathrm{SS}+\mathrm{SE})$ & 119 & 214 & 230 & 181 & 186.00 & 49.1 & 281 & 217 & 298 & 192 & 247.00 & 50.6 \\
\hline $\begin{array}{l}\text { Total of ASE in problem } \\
\text { and solution space }\end{array}$ & 267 & 307 & 341 & 258 & 293.25 & 38.3 & 314 & 250 & 334 & 221 & 279.75 & 53.1 \\
\hline
\end{tabular}

$\mathrm{T} 1$... T4 - Teams 1 to 4; $\quad$ Mean - average of all teams; $\quad \mathrm{SD}-$ standard derivation of all teams

The segments related to the design space have been analysed individually (as ASE design operations in the problem and the solution space) but also aggregated into two categories: (1) ASE and (2) problem-solution spaces. The aggregated analysis design operation combines problem and solution analysis (PA+SA), the aggregated synthesis design operation combines problem and solution synthesis (PS+SS), and the aggregated evaluation design operation combines problem and solution evaluation $(\mathrm{PE}+\mathrm{SE})$. Similarly, aggregated problem space design operations combine 
problem analysis, synthesis and evaluation (PA+PS+PE), and the aggregated solution space design operations combine solution analysis, synthesis and evaluation $(\mathrm{SA}+\mathrm{SS}+\mathrm{SE})$.

\subsection{Distribution of design operations}

For the purpose of focusing solely on ASE design operations within the problem and the solution space, the segments coded as other- and process-related discussion have been excluded from further analyses (frequency distribution and sequence analysis). Once the "process" and "other" segments were removed, the distribution of counted design operation segments (as presented in Table 3) was normalised in order to conduct further analyses using relative frequencies of design operations. Such normalisation implies that the sum of relative frequencies of all coded design operations segments equals $100 \%$. The resulting distribution of relative frequencies of design operation segments for each of the four teams during the two conceptual design activities is shown in Fig. 5. From this point on, only the relative frequencies of design operations and sequences of design operations are used. For the sake of simplicity, relative frequencies will be referred to as proportions.

Ideation activity

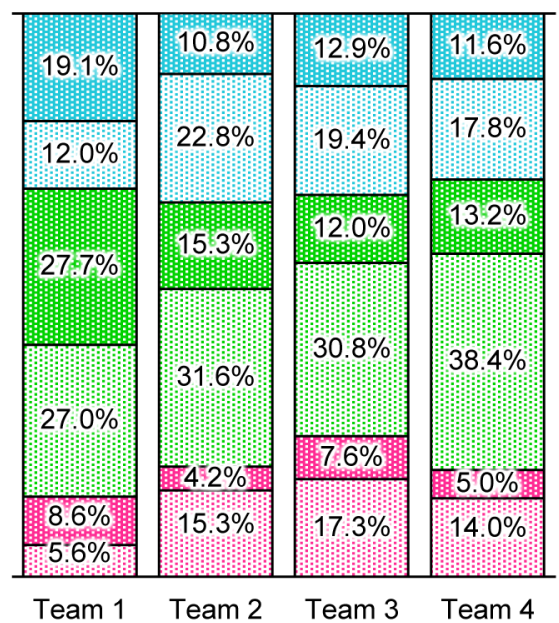

Concept review activity

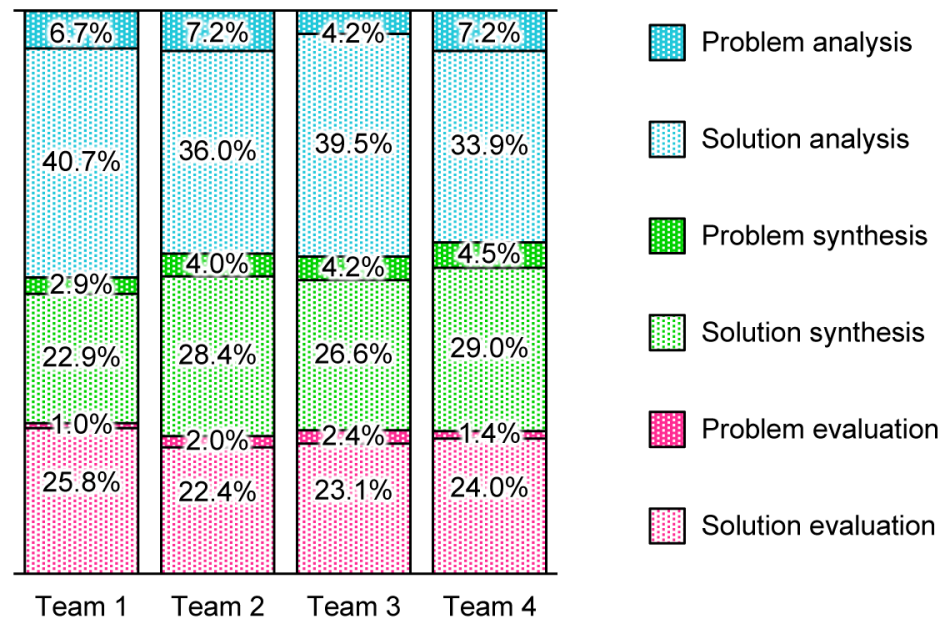

Fig. 5 Proportions of design operation segments during ideation and concept review activities for all teams

\subsubsection{Design operation distribution during ideation activity}

During the ideation activity, the most frequent ASE design operation in all four teams was synthesis (on average 49\% of all ASE design operations per team), followed by analysis (32\%), and evaluation (19\%) as the least frequent. Of all design operations, on average $37 \%$ were performed in the problem space, and $63 \%$ in solution space. One of the teams (Team 1 in Fig. 5) spent considerably more segments in the problem space (55\%) than the other three (30$33 \%$ ). On average, the most frequent design operation in problem space was problem synthesis (on average $46 \%$ of all design operations in problem space per team), followed by problem analysis (37\%) and problem evaluation (17\%). Similarly, the most frequent design operation in solution space was solution synthesis (on average $51 \%$ of all design operations in solution space per team), followed by solution analysis (28\%) and solution evaluation (21\%).

\subsubsection{Design operation distribution during concept review activity}

The descriptive statistics differ for the concept review activity, where the most frequent ASE design operation was analysis (on average $44 \%$ of all ASE design operations per team), followed by synthesis (31\%) and evaluation $(25 \%)$ as the least frequent. Of all design operations, on average $12 \%$ were performed in the problem space, and $88 \%$ in the solutions space, which is a considerable change compared to ideation. On average, the most frequent problem-space design operation was problem analysis (on average $53 \%$ of all design operations in problem space per team) followed by problem synthesis (33\%) and problem evaluation (14\%). The order concerning ASE is, again, the same in the solution space: solution analysis was the most frequent design operation in solution space (on average $43 \%$ of all design operations in solution space per team), followed by solution synthesis $(30 \%)$ and solution evaluation $(27 \%)$.

\subsubsection{Comparison of ideation and concept review activities}

A two-tailed paired-sample t-test was conducted to compare proportions of design operations separately and aggregated into both ASE and problem-solution space. Results of the test are given in Table 4 . The normality of design operation distribution has been assumed following a similar approach by Mc Neill et al. (1998). Despite the small sample of teams, significant differences have been identified for the two conceptual design activities. 
There is a significant difference in proportions of ASE design operations in problem and solution space for ideation and concept review activities. Conducting one-tailed paired-sample t-test reveals that the proportions of problem analysis, problem synthesis, problem evaluation and solution synthesis are significantly higher $(\mathrm{p}<0.05)$ during the ideation activity. In contrast, the frequencies of solution analysis and solution evaluation are significantly higher $(\mathrm{p}<0.05)$ during the concept review activity.

Table 4 T-test comparing proportions of design operations during ideation and concept review activities

\begin{tabular}{|c|c|c|c|c|c|c|}
\hline \multirow{2}{*}{ Code } & \multicolumn{2}{|c|}{ Ideation } & \multicolumn{2}{|c|}{ Concept review } & \multirow{2}{*}{ t value } & \multirow{2}{*}{$P$ value } \\
\hline & Mean (\%) & SD (\%) & Mean $(\%)$ & SD (\%) & & \\
\hline Problem analysis (PA) & 13.6 & 3.8 & 6.3 & 1.4 & 3.537 & $0.038^{*}$ \\
\hline Problem synthesis (PS) & 17.1 & 7.2 & 3.9 & 0.7 & 3.319 & $0.045^{*}$ \\
\hline Problem evaluation (PE) & 6.4 & 2.1 & 1.7 & 0.6 & 4.052 & $0.027 *$ \\
\hline Solution analysis (SA) & 18.0 & 4.5 & 37.6 & 3.1 & -5.778 & $0.010^{*}$ \\
\hline Solution synthesis (SS) & 31.9 & 4.7 & 26.7 & 2.7 & 3.661 & $0.035^{*}$ \\
\hline Solution evaluation (SE) & 13.0 & 5.1 & 23.8 & 1.5 & -3.297 & $0.046^{*}$ \\
\hline Analysis (PA+SA) & 31.6 & 1.7 & 43.9 & 2.6 & -8.593 & $0.003 * *$ \\
\hline Synthesis (PS+SS) & 49.0 & 5.2 & 30.6 & 3.4 & 4.932 & $0.016^{*}$ \\
\hline Evaluation $(\mathrm{PE}+\mathrm{SE})$ & 19.4 & 4.4 & 25.5 & 1.0 & -2.442 & 0.092 \\
\hline Problem space $(\mathrm{PA}+\mathrm{PS}+\mathrm{PE})$ & 37.0 & 12.3 & 11.9 & 1.5 & 3.751 & $0.033^{*}$ \\
\hline Solution space $(\mathrm{SA}+\mathrm{SS}+\mathrm{SE})$ & 63.0 & 12.3 & 88.1 & 1.5 & -3.751 & $0.033^{*}$ \\
\hline
\end{tabular}

$* \mathrm{p}<0.05 * * \mathrm{p}<0.01$

Table 4 also shows significant difference in proportions of design operations aggregated to ASE and problemsolution segments. Conducting one-tailed paired-sample t-test reveals that the proportion of analysis design operation is significantly higher $(\mathrm{p}<0.01)$ during the concept review activity, while the proportion of synthesis is significantly higher $(\mathrm{p}<0.01)$ during the ideation activity. Furthrmore, the proportion of problem-related discussion is significantly higher $(p<0.05)$ and the proportion of solution-related discussion is significantly lower $(p<0.05)$ during the ideation activity, when compared to concept review activity.

\subsection{Sequences of design operations}

The probabilities of one design operation following another design operation, i.e. one state transition following another state transition have been interpreted as probability matrices in Markov processes. The probability matrix (Markov matrix) is a right stochastic matrix - a square matrix used to describe the probabilities of moving from one element in the matrix (in this case a design operation) to all other elements (Gagniuc 2017). It is important to note that the common term used to link the elements of a probability matrix is a "transition". However, to reduce the ambiguity when discussing transitions between design operations (transitions of transitions in the proposed model), this term has been replaced with "moves".

For each of the teams, the total number of moves between pairs of design operations have been counted and entered into the corresponding cells of the matrix. The rows of the matrix were normalised to calculate the probabilities (the sum of values in each row of a right stochastic matrix is 1). Each of the resulting matrices represents the probability matrix for that particular team. Finally, in order to summarise the data, the probabilities matrices have been averaged per team. The resulting average probability matrices are shown in Table 5. Cells of the matrices have been coloured to facilitate identification of moves between design operations that are most likely to appear. During the ideation activity, the most probable design operation to come after problem analysis was problem synthesis (32.3\% probability), after problem synthesis it was problem analysis $(28.9 \%)$ and after problem evaluation it was also problem analysis $(33.8 \%)$. Furthermore, solution analysis was most likely to be followed by solution synthesis (43.1\%), solution synthesis by solution analysis (38.2\%), and solution evaluation by solution synthesis (42.3\%). As for the aggregated design operations, the most likely moves were as follows: analysis was most likely followed by synthesis $(58.3 \%)$, synthesis by synthesis $(40.5 \%)$ or analysis $(40.0 \%)$, and evaluation by synthesis $(54.9 \%)$.

During the concept review activity, the most likely moves starting with each of the ASE design operations in problem and solution space were as follows: problem analysis was most likely to be followed by solution synthesis (36.1\%), problem synthesis by solution synthesis $(34.8 \%)$, problem evaluation by solution analysis $(46.5 \%)$, solution analysis by solutions synthesis $(33.4 \%)$, solution synthesis by solution analysis $(52.4 \%)$, and solution evaluation by solution analysis (44.7\%). The most likely moves for each of the aggregated ASE design operations were as follows: analysis was most likely to be followed by synthesis $(38.8 \%)$, synthesis by analysis $(53.1 \%)$ and evaluation by analysis (53.4\%). Please consult Table 5 for the probabilities of moves between all pairs of ASE design operations. 
Table 5 Probability matrices for moves between ASE design operations in problem and solution space (left) and aggregated to ASE (right), obtained from ideation and concept review activities (both on average per team)

\begin{tabular}{|c|c|c|c|c|c|c|c|c|c|c|c|}
\hline \multicolumn{9}{|c|}{ ASE design operations in problem and solution space } & \multicolumn{3}{|c|}{ ASE design operations } \\
\hline \multirow{7}{*}{ 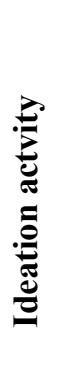 } & & PA & PS & $\mathbf{P E}$ & SA & SS & SE & & & & \\
\hline & PA & 0.134 & 0.323 & 0.192 & 0.013 & 0.301 & 0.037 & \multirow[b]{3}{*}{ Analysis } & \multirow[b]{2}{*}{ Analysis } & \multirow[b]{2}{*}{ Synthesis } & \multirow[b]{2}{*}{ Evaluation } \\
\hline & PS & 0.289 & 0.256 & 0.191 & 0.022 & 0.209 & 0.033 & & & & \\
\hline & PE & 0.338 & 0.288 & 0.040 & 0.048 & 0.267 & 0.019 & & 0.170 & 0.583 & 0.247 \\
\hline & SA & 0.079 & 0.130 & 0.007 & 0.109 & 0.431 & 0.244 & Synthesis & 0.400 & 0.405 & 0.195 \\
\hline & SS & 0.059 & 0.066 & 0.007 & 0.382 & 0.313 & 0.173 & Evaluation & 0.348 & 0.549 & 0.102 \\
\hline & SE & 0.083 & 0.118 & 0.000 & 0.260 & 0.423 & 0.115 & & & & \\
\hline 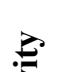 & & PA & PS & $\mathbf{P E}$ & SA & SS & SE & & & & \\
\hline : & PA & 0.071 & 0.056 & 0.081 & 0.312 & 0.361 & 0.118 & & & & \\
\hline $\bar{\pi}$ & PS & 0.204 & 0.000 & 0.191 & 0.123 & 0.348 & 0.134 & & Analysis & Synthesis & Evaluation \\
\hline . & $\overline{P E}$ & 0.194 & 0.000 & 0.000 & 0.465 & 0.094 & 0.248 & Analysis & 0.319 & 0.388 & 0.293 \\
\hline$\stackrel{0}{2}$ & SA & 0.046 & 0.048 & 0.004 & 0.261 & 0.334 & 0.308 & Synthesis & 0.531 & 0.179 & 0.290 \\
\hline 륭 & SS & 0.036 & 0.009 & 0.000 & 0.524 & 0.144 & 0.286 & Evaluation & 0.534 & 0.317 & 0.148 \\
\hline ể & SE & 0.079 & 0.067 & 0.015 & 0.447 & 0.262 & 0.130 & & & & \\
\hline
\end{tabular}

Probability matrix of an individual team can be multiplied with the team's corresponding proportion of design operations segments (presented in Fig. 5) to calculate proportions of particular moves between two design operations for that particular team. The obtained results correspond to the proportions of moves from one ASE design operation to another within the spaces (problem to problem and solution to solution) and in-between the spaces (problem to solution and solution to problem) in a single team. As part of the presented research, the proportion matrices have been averaged per teams in order to summarise the data (Table 10 in the Appendix). Moreover, the average proportion matrices have been mapped onto the state-transition model visualisation proposed in Fig. 4, by adjusting the thickness of the transition arrows. The resulting visualisations (Fig. 6) reflect the average proportional distribution of sequences of design operations throughout the ideation and the concept review activities.

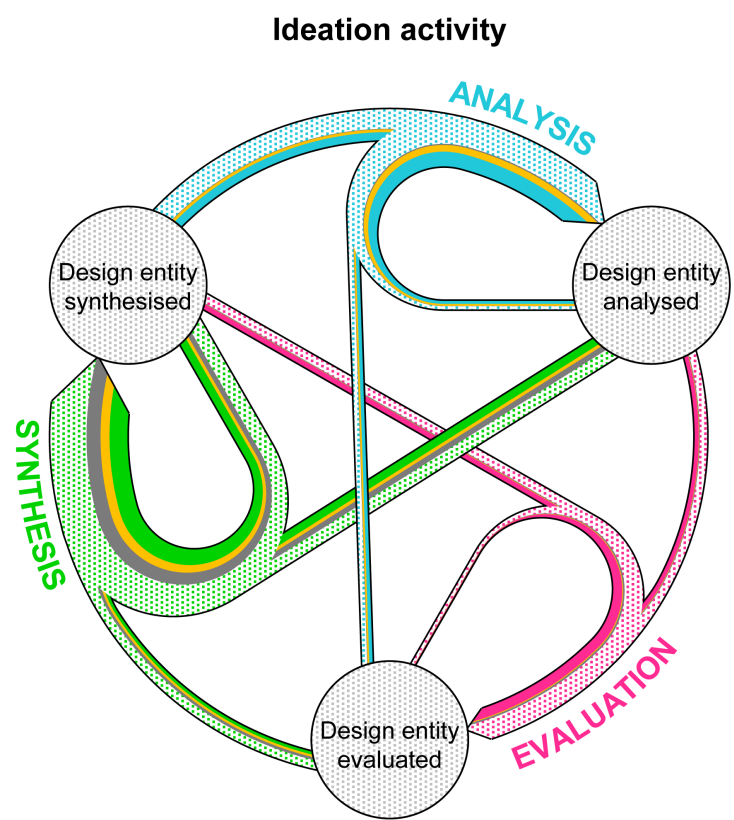

within solution space switch from problem to solution space
Concept review activity

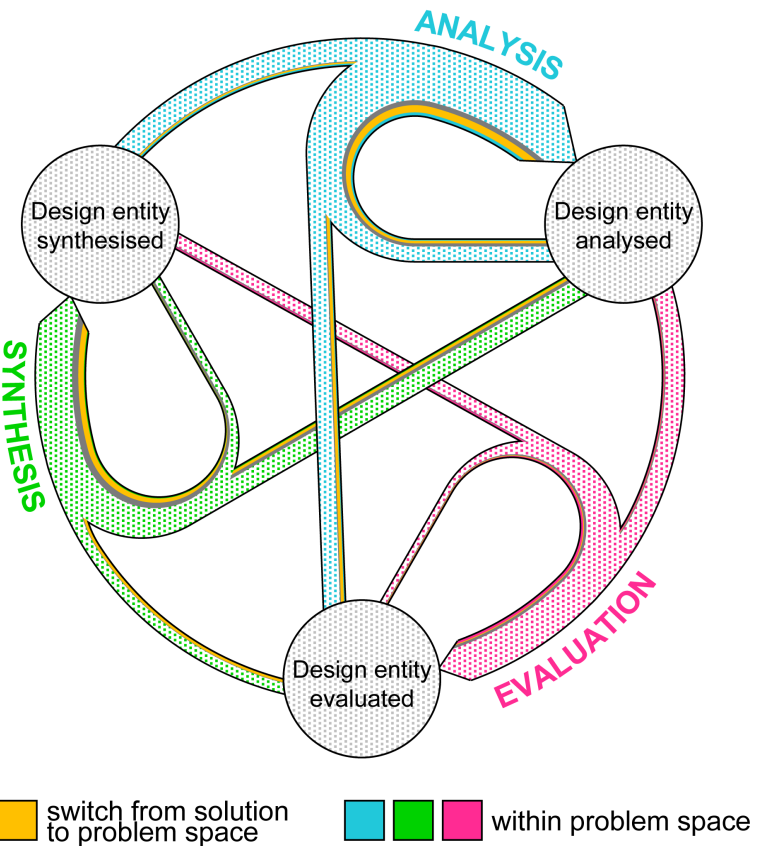

Fig. 6 State-transition visualization of the average proportions of sequences of ASE design operations within and in-between the problem and the solution space during ideation (left) and concept review (right) activity 
Two separate visualisations of state transitions have been developed to qualitatively compare the micro-scale design processes of teams engaged in ideation and concept review activities. Unlike the proportions of design operations presented in Table 4, the state-transition model visualisations provide additional insights on what design operations are likely to follow once a problem or solution entity has been analysed, synthesised or evaluated. Moreover, the overall thickness of the arrows entering the state nodes reflects the proportion of analysis, synthesis and evaluation during the activities. The visualisations provide qualitative insights into:

- Traces of ASE performed within the problem space (continuous evolution of the problem space)

- Traces of ASE performed within the solution space (continuous evolution of the solution space)

- Traces of ASE performed to switch from problem to solution space, and from solution to problem space (co-evolution of the problem and the solution space)

Finally, further analysis can be conducted by taking into account sequences of more than two subsequent design operations. Within the extent of presented study, the sequences of three design operations were counted and normalised for each of the teams, thus providing proportions of particular moves between three design operations. The resulting proportions were averaged across all teams (Table 11 in the Appendix). Sequences of multiple design operations should facilitate identification of patterns related to performing ASE design operations in the problem and the solution space. Nevertheless, mapping the proportions of sequences of three or more design operations onto the state-transition model results in visualisation identical to those shown in Fig. 6.

\subsubsection{State transition sequences during ideation activity}

The averaged proportions of moves between ASE design operations during the ideation activity (Fig. 6 on the left, based on Table 10) reveal several similarities in performing analysis, synthesis and evaluation within the problem and the solution space. For example, the most frequent sequences of two design operations within both spaces were synthesis to synthesis, synthesis to analysis and analysis to synthesis. The decreasing order of the remaining moves in both spaces was: synthesis to evaluation, analysis to evaluation, evaluation to analysis, analysis to analysis, and evaluation to evaluation. Nevertheless, the proportion of moves in problem and solution space differs largely in the case of the evaluation to synthesis sequence, which appeared primarily within the solution space. Examination of three subsequent design operations (Table 11) reveals the most frequent sequences within the problem space: synthesis - analysis - synthesis (on average $2.3 \%$ of all sequences) and synthesis - synthesis - synthesis $(2.2 \%)$; and within the solution space: synthesis - analysis - synthesis (5.9\%) and synthesis - synthesis - analysis (4.1\%).

Further insights can be derived from Table 10 and Fig. 6. Regarding the moves from one space to another, teams would switch from solution to problem space mainly to perform problem synthesis (on average $5.8 \%$ of all moves per team), and problem analysis (4.3\%). On average, the most frequent moves from solution to problem space were from solution analysis and solution synthesis to problem synthesis (both $2.1 \%$ ), followed by moves from solution synthesis to problem analysis $(1.8 \%)$ and solution evaluation to problem synthesis $(1.6 \%)$. Only a few instances have been identified where teams switched the space to evaluate a problem $(0.4 \%$ in total). As for the opposite direction, when switching to the solution space, teams did it primarily to synthesise solutions (on average $8.9 \%$ of all moves per team), and rather less frequently to evaluate $(1.1 \%)$ or analyse $(0.8 \%)$ solutions. Hence, problem analysis, synthesis and evaluation were all most likely to be followed by solution synthesis if space is switched.

Nevertheless, the probabilities of moves during the ideation activity (Table 5) show that once the teams switched from problem to solution space or vice versa, it was very likely that the next few transitions will remain in that space, before switching spaces again. Thus, the adding up of proportions of design operation moves presented in Table 10 shows that on average $52.4 \%$ of the moves took place within the solution space, $26.4 \%$ within the problem space, and $21.2 \%$ in-between the spaces.

The most frequent sequence of three design operations which led to switching from problem to solution space was: problem synthesis - problem analysis - solution synthesis (on average 1.3\% of all sequences). Similarly, the other way around it was: solution synthesis - solution analysis - problem synthesis (1.6\%). Please consult Table 11 for a detailed proportional overview for sequences of three design operations.

\subsubsection{State transition sequences during concept review activity}

The observed proportions of sequences of ASE design operations during concept review activity differ substantially in comparison to ideation (Fig 6 on the right, based on Table 10). For the most part, when the teams switched from solution to problem space during concept review, according to Table 5, it was unlikely that the next transitions would again be performed within the problem space. In contrast, when they switched from problem to solution space, it was likely for a larger number of solution-related design operations to follow. Thus, on average $79.5 \%$ of the design operation moves took place within the solution space and only $3.2 \%$ within the problem space, with $17.3 \%$ of moves in-between the problem and the solution space.

Consequently, as shown in Table 10 and Fig. 6, the most frequent sequences of two design operations during concept review appeared solely within the solution space. These are, in decreasing order: synthesis to analysis (on average $13.9 \%$ of all moves per team), analysis to synthesis $(12.6 \%)$, analysis to evaluation (11.6\%), evaluation to analysis $(10.6 \%)$ and analysis to analysis $(9.9 \%)$. The most frequent sequences within the problem space were from synthesis to analysis $(0.9 \%)$ and from synthesis to evaluation $(0.7 \%)$. No moves from synthesis to synthesis, 
evaluation to synthesis and evaluation to evaluation have been identified within the problem space. Interestingly, such moves were also the least frequent within the solution space.

Further examination reveals that the most frequent sequences of three design operations (Table 11) within the solution space were analysis - synthesis - analysis (on average $6.8 \%$ of all sequences), analysis - evaluation analysis (5.0\%), and synthesis - analysis - evaluation (4.9\%). As expected, due to the low proportion of problemrelated moves, no frequent sequences of three design operations within the problem space can be singled out.

Teams most frequently switched from solution to problem space in order to analyse existing problems (on average $4.6 \%$ of all moves per team) or to synthesise new ones (3.6\%). As shown in Table 10, these moves most often followed after solution evaluation and solution analysis. The other way around, teams frequently switched from problem space to solution space in order to perform solution synthesis $(3.9 \%)$. For example, both problem analysis and problem synthesis were most frequently followed by solution synthesis once space was switched.

The most frequent sequences of three design operations which led to switching from problem to solution space were: problem synthesis - problem evaluation - solution analysis ( $0.5 \%$ of all sequences), and problem synthesis problem analysis - solution synthesis $(0.4 \%)$. The most frequent sequence from solution to problem space was solution analysis - solution evaluation - problem analysis ( $0.9 \%$, see Table 11 for a detailed overview of sequences).

\subsubsection{Differences in state transition sequences between ideation and concept review activities}

The significant differences in the probabilities of moves between design operations during ideation and concept review activities have been identified by performing a two-tailed paired-sample t-test on the probability matrices derived for each team (note that Table 5 shows only the mean values of probability matrices for all teams). Due to a relatively large number (36) of possible sequences of two design operations, only the sequences of significantly different probabilities are shown in Table 6.

Table 6 T-test comparing probabilities of design operation moves during ideation and concept review activities

\begin{tabular}{|c|c|c|c|c|c|c|}
\hline \multirow{2}{*}{$\begin{array}{l}\text { Design operation } \\
\text { sequence }\end{array}$} & \multicolumn{2}{|c|}{ Ideation } & \multicolumn{2}{|c|}{ Concept review } & \multirow{2}{*}{ t value } & \multirow{2}{*}{$P$ value } \\
\hline & Mean & SD & Mean & SD & & \\
\hline $\mathrm{PA} \rightarrow \mathrm{PS}$ & 0.323 & 0.149 & 0.056 & 0.041 & -3.438 & $0.022 *$ \\
\hline $\mathrm{PA} \rightarrow \mathrm{PE}$ & 0.192 & 0.055 & 0.081 & 0.063 & -2.649 & $0.034^{*}$ \\
\hline $\mathrm{PA} \rightarrow \mathrm{SA}$ & 0.013 & 0.016 & 0.312 & 0.130 & 4.559 & $0.023^{*}$ \\
\hline $\mathrm{PS} \rightarrow \mathrm{PS}$ & 0.256 & 0.080 & 0.000 & 0.000 & -6.426 & $0.008^{* *}$ \\
\hline $\mathrm{PE} \rightarrow \mathrm{PS}$ & 0.288 & 0.038 & 0.000 & 0.000 & -15.163 & $0.001 * *$ \\
\hline $\mathrm{PE} \rightarrow \mathrm{SE}$ & 0.019 & 0.038 & 0.248 & 0.103 & 4.150 & $0.032 *$ \\
\hline $\mathrm{SA} \rightarrow \mathrm{PA}$ & 0.079 & 0.016 & 0.046 & 0.017 & -2.824 & $0.030^{*}$ \\
\hline $\mathrm{SA} \rightarrow \mathrm{SA}$ & 0.109 & 0.035 & 0.261 & 0.032 & 6.487 & $0.005^{* *}$ \\
\hline $\mathrm{SS} \rightarrow \mathrm{PS}$ & 0.066 & 0.012 & 0.009 & 0.011 & -7.003 & $0.012^{*}$ \\
\hline $\mathrm{SS} \rightarrow \mathrm{SE}$ & 0.173 & 0.029 & 0.286 & 0.069 & 3.031 & $0.037^{*}$ \\
\hline $\mathrm{SE} \rightarrow \mathrm{SA}$ & 0.260 & 0.110 & 0.447 & 0.129 & 2.195 & $0.025^{*}$ \\
\hline Analysis $\rightarrow$ Analysis & 0.170 & 0.026 & 0.319 & 0.006 & 11.338 & $0.001^{*}$ \\
\hline Analysis $\rightarrow$ Synthesis & 0.583 & 0.102 & 0.388 & 0.020 & -3.747 & $0.049^{*}$ \\
\hline Synthesis $\rightarrow$ Analysis & 0.400 & 0.030 & 0.531 & 0.047 & 4.728 & $0.038^{*}$ \\
\hline Synthesis $\rightarrow$ Synthesis & 0.405 & 0.047 & 0.179 & 0.097 & -4.194 & $0.036^{*}$ \\
\hline Synthesis $\rightarrow$ Evaluation & 0.195 & 0.026 & 0.290 & 0.057 & 3.040 & 0.050 \\
\hline Evaluation $\rightarrow$ Analysis & 0.348 & 0.057 & 0.534 & 0.094 & 3.404 & $0.003^{* *}$ \\
\hline Evaluation $\rightarrow$ Synthesis & 0.549 & 0.076 & 0.317 & 0.106 & -3.571 & $0.003 * *$ \\
\hline
\end{tabular}

$* \mathrm{p}<0.05 \quad * * \mathrm{p}<0.01$

Out of 36 possible sequences of two design operations, 11 have been found to significantly differ in their probability during the ideation and the concept review activity (Table 6). One-tailed paired-sample t-test further reveals that the probabilities of design operation sequences directed towards the problem space (problem analysis to problem synthesis, problem analysis to problem evaluation, problem synthesis to problem synthesis, problem evaluation to problem synthesis, solution analysis to problem analysis and solution synthesis to problem synthesis) are significantly higher $(p<0.05)$ during the ideation activity. Moreover, the probabilities of design operation sequences towards the solution space (problem analysis to solution analysis, problem evaluation to solution evaluation, solution analysis to solution 
analysis, solution synthesis to solution evaluation and solution evaluation to solution analysis) are significantly higher $(p<0.05)$ during the concept review activity. As for the transitions aggregated into ASE, the probabilities of moves from analysis to synthesis, synthesis to synthesis and evaluation to synthesis are significantly higher $(\mathrm{p}<0.05)$ during the ideation activity, while the probabilities of moves from analysis to analysis, synthesis to analysis and synthesis to evaluation are significantly higher $(\mathrm{p}<0.05)$ during the concept review activity.

\section{Discussion}

The presented study aims to contribute to the research of the team design process in two ways. The first is providing a state-transition model of team conceptual design activity, which reflects the micro-scale sequences of analysis, synthesis and evaluation within and in-between the problem and the solution space. The main advantage of the proposed state-transition model is its ability to map various sequences of ASE design operations which emerge during team conceptual design activities. The second contribution to the research of team designing stems from applying the developed model to identify patterns of ASE design operations which design teams perform during ideation and concept review activities. The use of the model facilitated identification of both the activity-specific patterns and patterns that are likely to appear during both activities. The identified patterns are discussed by addressing the two research questions in the following subsections, before a final reflection on the model's utility is made.

\subsection{Patterns of ASE design operations within the problem-solution space}

Although the proportions of segments related to discussions of problems versus solutions space alter across the four teams (problem-solution focus of teams can vary as shown by Jiang et al. 2014), there exist similarities in proportions of sequences of executing ASE design operations within these two dimensions of design space. This similarity can primarily be explored during ideation, where, according to Table 4, teams spent significantly more segments discussing the problem space. Qualitatively, the order of most likely moves between ASE design operations (Table 5) is consistent when considering sequences within the problem and sequences within the solution space. During ideation, synthesis is in both spaces most likely to be followed by analysis and least likely by evaluation. During concept review, in both of the spaces synthesis is also most likely to be followed by analysis and least likely by another synthesis design operation. Additionally, the most likely design operation to follow analysis during ideation was synthesis, and the least likely was analysis. On the other hand, the most likely move from evaluation during concept review was to analysis, again regardless of the dimension of the design space. These results can be related to the "find and modify" patterns of ideation, which Sarkar and Chakrabarti $(2014,2017)$ identified within both the problem and the solution space.

By analysing the sequential strings of coded segments for each of the teams and the corresponding probabilities and proportions of moves between design operations, it is possible to examine the fine-grain patterns in teams' design processes. In Fig. 7 the state-transition model visualisation is used to illustrate three common patterns of ASE design operation sequences obtained for both the ideation and the concept review activity. These patterns are conceptualised as templates on which sequences of several design operations can be mapped to indicate common micro-scale building blocks of the team conceptual design process. The patterns were initially identified within the strings of protocol codes, as sequences of coded segments which are articulated due to their repetition. The identified patterns have then been further investigated by mapping the probabilities of moves between design operations (Table 5) and proportions of these particular sequences available in Tables 10 and 11.

a)

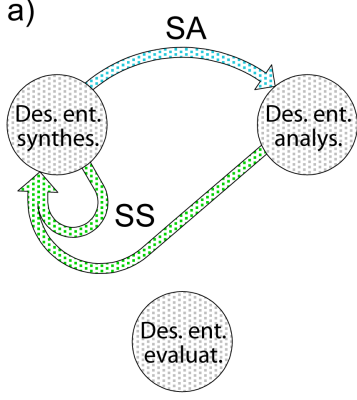

b)

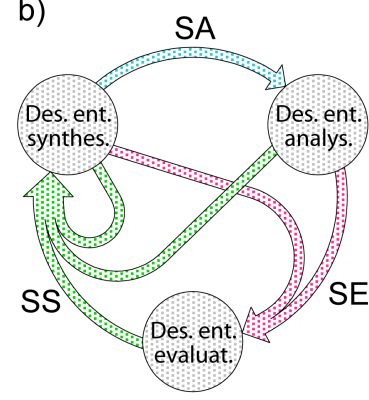

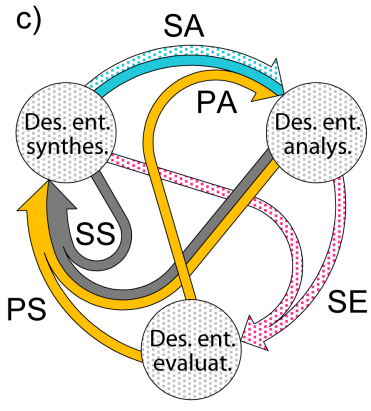

SA
Fig. 7 Common patterns of state transitions sequences during team conceptual design activities: a) cycles of solution synthesis and analysis; b) sequences of solution synthesis, analysis and evaluation; c) synthesis as means of switching in-between problem and solution space

The first pattern present in both activities (Fig. 7a) comprises the reciprocating cycles of solution synthesis frequently intercepted by solution analysis. Moves in-between solution synthesis and analysis have been shown as the most probable during both activities (Table 5). Moreover, adding up the proportions of state transitions reveals that $57.1 \%$ of sequences of two (Table 10 ), and $42.3 \%$ of sequences of three (Table 11) solution-related design operations during ideation can be mapped onto this pattern. As for concept review, the pattern includes $38.4 \%$ of sequences of two and $22.1 \%$ of three solution-related design operations. 
Thes cycles forming the first identified pattern have already been given attention in the literature. For example, Smith and Tjandra (1998) interpreted analysis-synthesis cycles as iteration in design activity. They state that the iteration intensifies as teams progress in the conceptual design phase. A similar interpretation has been provided by Sung and Kelley (2018), who described the phenomenon as a bi-directional iteration of designing solutions and predicting possible consequences of the solution ideas. Furthermore, Sauder and Jin (2016) have observed that questioning and clarification of design solutions (solution analysis) stimulates generative thinking processes which in return trigger generative (solution synthesis) design operations. Similarly, Cardoso et al. (2016) interpreted questions as drivers of discourse in design team ideation activity. According to these studies, the analysis of the shared design space appears to be an important driver of stimulation responsible for the generative (synthesis) processes. Finally, the dominance of solution synthesis within the cycle can be characterised as a decoupled ideation (Cash and Storga 2015), where solution ideas are appearing without the need of switching to problem space and triggering the co-evolution episodes. The presented data thus support the claim that the alternation of synthesis and analysis in both the problem and the solution space is typical for conceptual design activities (Liikkanen and Perttula 2009; Afacan and Demirkan 2011). An excerpt of the experiment session transcripts which demonstrates the first pattern is available in Table 7.

Table 7 An excerpt of team discussion demonstrating the reciprocating cycles of solution synthesis and analysis

\begin{tabular}{|c|c|c|}
\hline Participant & Segment transcript & Code \\
\hline $\mathrm{P} 1$ & $\begin{array}{l}\text { [discussing attachment to the balloon] } \\
\text { We have to minimise the mass. }\end{array}$ & PS \\
\hline $\mathrm{P} 2$ & We could use welding. & SS \\
\hline $\mathrm{P} 2$ & Or... Uhm. Glue, adhesive. & SS \\
\hline $\begin{array}{l}\mathrm{P} 3 \\
\mathrm{P} 2\end{array}$ & $\begin{array}{l}\text { Adhesive? I guess we can call it both (glue and adhesive). } \\
\text { Yes. }\end{array}$ & SA \\
\hline P3 & We also have Velcro. & SS \\
\hline $\mathrm{P} 2$ & You can put up magnetic. & SS \\
\hline $\mathrm{P} 2$ & [gesturing] Magnetic touch. I'm not sure how, but... & SA \\
\hline P3 & So, I guess we expand them now? & PROC \\
\hline $\begin{array}{l}\mathrm{P} 3 \\
\mathrm{P} 2\end{array}$ & $\begin{array}{l}\text { I got one actually, like a... [gesturing] I think is metal, like a flexible... } \\
\text { Yeah, the Gorillapod thing. }\end{array}$ & SS \\
\hline $\begin{array}{l}\mathrm{P} 3 \\
\mathrm{P} 2\end{array}$ & $\begin{array}{l}\text { Is that what it's called? } \\
\text { Yes. It's TP, thermoplastic. }\end{array}$ & SA \\
\hline $\begin{array}{l}\text { P1 } \\
\text { P2 }\end{array}$ & $\begin{array}{l}\text {... } \\
\text { [discussing which problem should be addressed next] } \\
\text { I think that camera movement is most important. } \\
\text { OK. }\end{array}$ & $\mathrm{PE}$ \\
\hline $\mathrm{P} 2$ & $\begin{array}{l}\text { If we want to move the camera mount from the base, I can think of one which has } \\
\text { two sets of drills, rotation axes. }\end{array}$ & SS \\
\hline $\mathrm{P} 2$ & $\begin{array}{l}\text { [gesturing] One in this direction, and the other one in this direction. I don't know } \\
\text { if you understood what I wanted to say? }\end{array}$ & SA \\
\hline $\mathrm{P} 1$ & Yes. & \\
\hline $\mathrm{P} 1$ & You could have it on a ball. & SS \\
\hline $\begin{array}{l}\mathrm{P} 2 \\
\mathrm{P} 2\end{array}$ & $\begin{array}{l}\text { Oh, and then manipulate the ball? } \\
\text { Yes, like a gyroscope or something. }\end{array}$ & SA \\
\hline $\begin{array}{l}\mathrm{P} 2 \\
\mathrm{P} 1\end{array}$ & $\begin{array}{l}\text { Can you sketch it? } \\
\text { [laughs] Not really. }\end{array}$ & PROC \\
\hline P3 & Maybe driven by gears to control the angle. & SS \\
\hline $\mathrm{P} 1$ & $\begin{array}{l}\text { You could have it... It does not have to be absolute any angle, because you could } \\
\text { have like [gestures] } 30 \text { degrees - } 30 \text { degrees - } 30 \text { degrees. }\end{array}$ & $\mathrm{SS}$ \\
\hline $\begin{array}{l}\text { P3 } \\
\text { P1 }\end{array}$ & $\begin{array}{l}\text { Oh, distinct angles. Not continuous. } \\
\text { Yes, distinct angles. }\end{array}$ & SA \\
\hline
\end{tabular}


The second pattern of design operations (Fig. 7b) identified within both activities includes sequences of solution synthesis, analysis and evaluation. This pattern builds on the first (divergent) pattern by incorporating solution evaluation as a converging operation. According to Tables 10 and 11, the summed-up proportions of state transitions included within the pattern account for $86.8 \%$ sequences of two, and $76.5 \%$ sequences of three solutionrelated design operations during ideation. Likewise, the pattern comprises $70.4 \%$ of sequences of two, and $51.1 \%$ sequences of three solution-related design operations during concept review activity.

The described sequences resemble the two types of thinking processes identified by Stempfle and Badke-Schaub (2002), where synthesised solutions are either immediately evaluated (process 1), or first analysed and then evaluated (process 2). If the synthesised solution is discarded, a new idea will be sought (Stempfle and BadkeSchaub 2002). A similar pattern can be described within the FBS framework, where a synthesised structure is first analysed to understand its behaviour, and then evaluated by comparing its behaviour to the expected behaviour (Gero and Kannengiesser 2014). An excerpt of the experiment session transcripts which demonstrates the second pattern is available in Table 8 .

Table 8 An excerpt of team discussion demonstrating the sequences of solution synthesis, analysis and evaluation

\begin{tabular}{|c|c|c|}
\hline Participant & Segment transcript & Code \\
\hline $\mathrm{P} 1$ & $\begin{array}{l}\text { [discussing alternatives to the universal screw camera attachment] } \\
\text { Duck tape - that is an idea. }\end{array}$ & SS \\
\hline $\mathrm{P} 1$ & I mean, it's not very usable, but... & SE \\
\hline $\mathrm{P} 1$ & We can use bands. Just bands. & SS \\
\hline $\mathrm{P} 2$ & Velcro? & $\mathrm{SS}$ \\
\hline $\mathrm{P} 1$ & Velcro. It can be sticked to the back of the camera. & \multirow{2}{*}{ SA } \\
\hline $\mathrm{P} 2$ & Velcro works in space actually. & \\
\hline P3 & I think it works in space because there are no forces in vacuum. & SE \\
\hline $\mathrm{P} 1$ & But you could use it to connect... Like a pouch. & SS \\
\hline P3 & I'm not saying its a bad idea. & SE \\
\hline P3 & A bag? Bag / kangaroo pouch. [laughs] & SS \\
\hline $\mathrm{P} 1$ & $\begin{array}{l}\text { The thing is, it's annoying if you have to screw your camera into something that is } \\
\text { fixed, especially to a balloon... }\end{array}$ & SE \\
\hline P3 & [interrupts and gestures] A clip in. & SS \\
\hline P3 & Which can go [points to written categories] here? & \multirow{2}{*}{ SA } \\
\hline $\mathrm{P} 1$ & Yes. & \\
\hline
\end{tabular}

The third identified pattern (Fig. 7c) indicates co-evolution of the problem and the solution space by combining state transitions which result in switching in-between problems and solutions by means of synthesis design operation. State transitions which can be mapped onto the third pattern account for $56.6 \%$ of sequences of two (Table 10), and $30.8 \%$ of three consecutive design operations (Table 11) in-between the problem and the solution space during ideation. If only sequences resulting in synthesis (as an indication of co-evolution) are considered, these percentages increase to $73.8 \%$ and $44.8 \%$. As for concept review, $51.6 \%$ of sequences of any two, and $26.0 \%$ of any three consecutive design operations in-between the problem and the solution space can be mapped. Again, the percentages are higher (93.7\% and 60.9\%) if only transitions resulting in synthesis are considered.

Once a synthesised solution entity is analysed or evaluated, a new problem is sometimes immediately discovered (synthesised) by the team members. As soon as the team develops a shared understanding of the new problem, they propose (synthesise) new solutions to the problem. In such co-evolution episode, the teams switch from solution to problem space and return to solution space. The new solution entity can again be further analysed and evaluated which can result in the identification of new problems. Such a pattern can be classified as a necessary part of refinement, a stereotype of progressive iteration as defined by Wynn and Eckert (2017). As the solution design goes through several levels of abstraction, each level can result in a new set of requirements (Srinivasan and Chakrabarti 2010a), so the solution undergoes iterative refinement until evaluated as satisfying. The described iterative pattern also corresponds to what Cash and Štorga (2015) define as integrated and iterative ideation since new solution ideas trigger new problems and vice versa. An excerpt of the experiment session transcripts which demonstrates the third pattern is available in Table 9. 
Table 9 An excerpt of team discussion demonstrating synthesis as means of switching in-between problem and solution space

\begin{tabular}{|c|c|c|}
\hline Participant & Segment transcript & Code \\
\hline $\mathrm{P} 1$ & I try to work out if we can get away with fewer motors than two. & PS \\
\hline $\mathrm{P} 2$ & [points to previously discussed solution] Exactly that's what I got over here. & SE \\
\hline $\mathrm{P} 1$ & $\begin{array}{l}\text { Yeah. But I don't know, because if you wanted it pushing down and think 'Oh yes, } \\
\text { I wanted that angle'. But it has only reached that far down and not... [points to } \\
\text { solution] }\end{array}$ & SA \\
\hline $\mathrm{P} 1$ & Then you obviously can't use the exact rotation. & SE \\
\hline $\mathrm{P} 1$ & $\begin{array}{l}\text { I think when you've got two degrees of freedom like that [points to solution] you } \\
\text { need two... sort of... at least two motors. }\end{array}$ & PS \\
\hline $\mathrm{P} 2$ & What if you had a really tight spiral and it gradually taper it to a triangle? & SS \\
\hline $\mathrm{P} 1$ & Yes, so it's going through every conceivable thing just using one motor? & \multirow{2}{*}{ SA } \\
\hline $\mathrm{P} 2$ & Yeah. & \\
\hline & $\ldots$ & \\
\hline $\mathrm{P} 1$ & $\begin{array}{l}\text { Could we just have like a... Plastic... [starts sketching] So it will be like that. And } \\
\text { then we put it in. And then just have... It's like a plastic... Where you just push it, } \\
\text { so it doesn't come out? }\end{array}$ & SS \\
\hline $\mathrm{P} 2$ & Yeah, like a clip. & \multirow[b]{2}{*}{ SA } \\
\hline $\mathrm{P} 1$ & $\begin{array}{l}\text { Yeah, so instead of a locking mechanism. You can do it with one hand. Just push } \\
\text { the latch and take it out, pull it out. }\end{array}$ & \\
\hline $\mathrm{P} 1$ & That would really simplify it. & $\mathrm{SE}$ \\
\hline $\mathrm{P} 2$ & $\begin{array}{l}\text { I mean, my only concern with that is in case the latch came undone, because } \\
\text { there's no spring holding it there. But in between somewhere of doing that } \\
\text { [pointing to solution] and making sure it didn't come under... }\end{array}$ & PS \\
\hline P3 & $\begin{array}{l}\text { What about a screw tightener thing... So that you have a notch inside... [starts } \\
\text { sketching] You have your plate slit in, a little notch in the base thing. And you } \\
\text { have a screw which just went in there. }\end{array}$ & SS \\
\hline $\mathrm{P} 2$ & So, it would be like a quarter inch screw and then just... [gestures] & \multirow{2}{*}{ SA } \\
\hline P3 & Yeah. & \\
\hline
\end{tabular}

\subsection{Differences between the ideation and the concept review activity}

The distribution of the coded design operations segments during the two types of conceptual design activity (Table 3 and Fig. 5) and the corresponding t-tests (Tables 4 and 6) reveal that teams are likely to exhibit different proportions of ASE design operations when performing different types of conceptual design activities. The comparison of ideation and concept review has revealed that the proportion of all six coded design operations differs significantly for these two types of activities studied. Based on these findings, it is argued that the activityspecific probabilities of ASE design operations appearing within and in-between the problem and the solution space can be utilised to investigate and model the change in state of the product being designed and change in state of the design process as defined by Reymen et al. (2006).

According to Table 5, teams exhibited significantly more problem-related discussion and solution synthesis during the ideation activity and significantly more solution analysis and evaluation during the concept review activity. Since ideation was the first collaborative activity of the team, it was natural for them to seek a shared understanding of the problem (Cross and Clayburn Cross 1995). Moreover, the decrease of design operations in problem space (especially problem synthesis) can be related to the drop in new requirements appearing towards the end of the conceptual design phase, as identified by Chakrabarti et al. (2004). During the concept review activity, the teams were more familiar with the design problem (space). Such a trend is qualitatively aligned with the findings of Jiang et al. (2014) and Gero et al. (2013), which imply the decrease in the proportion of problem-related issues as the conceptual design progresses. Additionaly, Gero and Jiang (2016) conclude that the concept review activities seem to be more solution-focused than the designing (ideation) sessions.

Ideation is often characterised as a divergent activity, considering that the generative design operation (synthesis) dominates the convergent one (evaluation) (Goldschmidt 2016). The fact that synthesis was the most frequent design operation for all of the studied teams during ideation session favours such characterisation. Furthermore, the proportions of ASE design operations (Fig. 5) correspond to the average proportions of the equivalent processes 
(within solution space) reported for the ideation activities in Gero et al. (2013). Their study suggests that these proportions are also affected by the type of the ideation method used. For example, the protocol study of brainstorming sessions presented in Kan et al. (2011) shows a somewhat higher rate of synthesis design operation, mainly in the solution space. It can thus be argued that the application of design and creativity methods during the conceptual design activity will likely affect the fine-grain patterns of the design process.

On the other hand, the protocol analysis of the concept review activity has revealed significantly higher proportions of solution-related discussion, particularly manifested in higher proportions of solution analysis and evaluation design operations. The studies of conceptual design where the design brief instructed the proposal of a single concept solution (which had to converge) suggest that engineering design teams will most frequently perform solution analysis and solution evaluation design operations (solution analysis and problem clarification in Casakin and Badke-Schaub (2015) and content analysis in Stempfle and Badke-Schaub (2002), followed by solution evaluation). Moreover, despite the increase in solution-related discussion, the proportion of solution synthesis is significantly lower when compared to ideation, thus providing additional justification for describing concept review as a mainly a convergent activity.

If the two activities are compared with the stereotypes of progressive iteration (Wynn and Eckert 2017), a link can be found between ideation and exploration (divergence) stereotype, and between concept review and convergence stereotype. Wynn and Eckert (2017) describe exploration as concurrent and iterative initial development of the problem and the solution, where the ill-defined nature of design goals is emphasised. Such progressive iteration is reflected in the evolution and co-evolution of the problem space during ideation, as shown in Fig. 8. Convergence is described as an iterative adjustment towards a satisfying goal, once the main form of the design has been determined at a certain level of definition (Wynn and Eckert 2017). During concept review, the designers would select and synthesise the most promising concept and would then iteratively refine different aspects of the final solution proposal.

The comparison of the two activities has revealed not only the different proportions of design operations (Table 4) but also the activity-specific sequences of ASE design operations. The activity-specific sequences represent the moves between two design operations whose probability changed significantly between the two types of activities (Table 6). The sequences with significantly higher probabilities during ideation and concept review activities have been illustrated as state transitions in Fig. 8.

Ideation activity

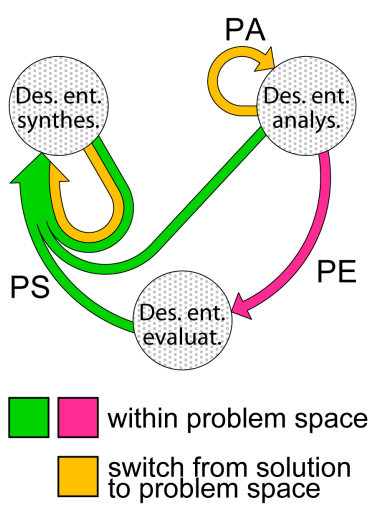

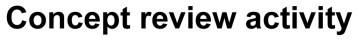

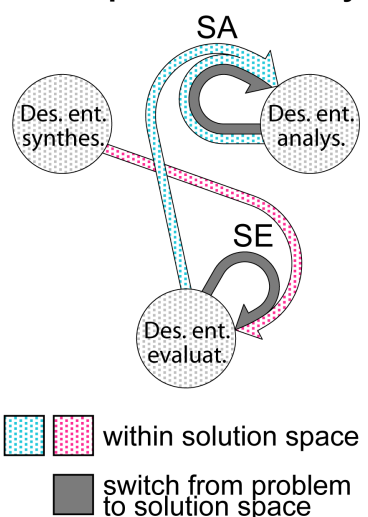

Fig. 8 State transitions distinctive for the ideation (left) and to the concept review activity (right)

The significant changes in the proportions of design operation sequences identified in Table 6 and Fig. 8 again point out the divergent features of the ideation and convergent features of the concept review activity. As described, the divergent alternation of solution synthesis and analysis (Fig. 7a) accounts for almost $60 \%$ of solution-related discussions during ideation. However, as shown in Fig. 8, the divergent features of ideation are also reflected in higher proportions of synthesis moves within the problem space, but also in-between the problem and the solution space. On the other hand, convergent cycles during the concept review activity are characterised by the sequences of analysis and evaluation design operations performed as part of developing and refining the final proposal of the conceptual solution (Fig. 7b). As shown in Fig. 8, the probability of evaluating a synthesised solution is significantly higher during concept review. Furthermore, the evaluated solutions are more likely to be repeatedly analysed. Here, the analysis design operation is essential for the better understanding of team members, and leads to progress in team design activities, whether it is used as clarification (Casakin and Badke-Schaub 2015; 2017), or questioning (Cardoso et al. 2016). Hence, achieving common ground (understanding), as highlighted by Hultén et al. (2018), appears to be an important ingredient of a team's creative process during conceptual design activities.

Finally, the protocol analysis provided an insight into teams' practices of using ASE design operations to switch from problem to solution space and vice-versa. An interesting finding is that moves from problem to solution space 
are performed mainly to synthesise new entities, while moves from solution to problem space appear either because a new problem was identified, or the focus is again set to the analysis of the existing problem entities. Such patterns support the concept of problem-solution co-evolution as described in studies by Dorst and Cross (2001) and Visser (2009). Therefore, the moves in-between the problem and the solution space which result in the synthesis of design entities can be characterised as identifiers of the likely co-evolution episodes.

Although studies have reported co-evolution during both ideation and concept review activities (e.g. Toh and Miller 2015), there have been no clear insights on how the rate of co-evolution changes with the progress of conceptual design activity. The presented study shows moderately lower probabilities and frequencies of switching the space by performing synthesis design operation during the concept review activity, as opposed to ideation (moves from problem space to solution synthesis and from solution space to problem synthesis in Tables 5 and 10). Wiltschnig et al. (2013) who analysed the phenomena of co-evolution during several conceptual design meetings have identified that most of the co-evolution episodes imply new solution entities, rather than new problem entities. Similar insights can be drawn in this study, since the moves from problem space to solution synthesis, which are characteristic for such co-evolution episodes have been more frequent than moves from solution space to problem synthesis, during both of the activity types (as shown in Table 10 and Fig. 6). Nevertheless, while this was indeed the most likely space-switching scenario, the probabilities of space-switching moves which imply synthesis of problem entities (moves from solution space to problem synthesis in Table 5) must not be neglected, particularly during concept review. Namely, the convergent design activities are focused on evaluating solutions rather than creating new ones. The evaluation design operation has been defined in a way that it can implicitly reveal decomposed problems (Liikkanen and Perttula 2009). Such problem decomposition is argued to be the main reason why problem synthesis design operations are likely to follow solution evaluation if the space is switched. Also, Wiltschnig et al. (2013) reported that requirement analysis (problem analysis) is likely to trigger most of the co-evolution episodes, resulting in solution attempts (solution synthesis). The presented study shows that problem analysis certainly plays a valuable role in co-evolution during both ideation and concept review, expressed in the high probability of solution synthesis following problem analysis (Table 5). However, it was found that problem synthesis is more likely to be preceded by solution analysis and evaluation rather than solution synthesis when co-evolution occurs (as seen in Table 5 and Fig. 8c).

\subsection{Reflection on the state-transition model}

The benefit of adapting the definitions of ASE design operations built into the state-transition model manifests mainly in the proportions of solution analysis, problem synthesis and problem evaluation design operations. Firstly, the literature review revealed that analysis is often conceptualised only as a problem-clarification step in the design process (e.g. Roozenburg and Eekels 1995; Mc Neill et al. 1998, Wodehouse and Ion 2010; Fiorineschi et al. 2016). However, the high proportions of solution analysis design operation reveal that teams spent a considerable portion of conceptual design activity increasing the understanding of design solutions. The model, therefore, exhibits the critical role of analysis as a design operation performed in both the problem and the solution space. For that reason, it can be argued that both problem and solution analysis should be traced as independent fine-grain steps in the conceptual design process, where the individual and shared understanding are increased by means of questioning and clarification, rather than being incorporated as part of the evaluation step (see, e.g. Mc Neil et al. 1998, Srinivasan and Chakrabarti 2010a).

Secondly, the reviewed fine-grain models of designing articulate mainly the synthesis of new solution entities during the design activity (e.g. Gero 1990; Stempfle and Badke-Schaub 2002). Nevertheless, high proportions of problem synthesis design operation, especially during ideation, indicate that new problem entities are also appearing repeatedly throughout the team conceptual design activity. For example, ideation activities have shown highly probable cycles of problem synthesis design operations. Hence, by capturing the synthesis of design entities in both dimensions of the design space, the proposed model complements the existing descriptive research efforts. Finally, although neglected in some models of the design process, the problem evaluation design operation accounts for about $6 \%$ of all design operations during ideation, and $2 \%$ during concept review activity. While this is a relatively small proportion, it shows that teams evaluate not only the proposed solutions but also the design entities within the problem space (e.g. requirement prioritisation and constraint assessment).

The state-transition model's ability to capture sequences of any pair of observable design operations (including the repeating cycles of a single design operation) has resulted in representations of the team conceptual design activity which could not be replicated by other descriptive models of designing. Particularly, many of the observed patterns during ideation and concept related activity (e.g. alternation of analysis and synthesis, and the repetitive cycles of synthesis or analysis) cannot be mapped directly on the reviewed design models. For example, the synthesis operation in the FBS framework can be followed by analysis (Gero and Kannengiesser 2014). However, according to the FBS ontology coding scheme (Gero et al. 2013), synthesis can follow analysis only if evaluation appears inbetween them. And while such transitions might be the case at a cognitive design level or for individual designing, the reported study showed that there are many observable episodes of analysis-synthesis moves appearing during team ideation and concept review. The same can be argued for synthesis-synthesis sequences occurring during ideation, and analysis-analysis and synthesis-evaluation sequences occurring during concept review. 
Moreover, not all of the observed ASE patterns can be identically mapped onto the model of thinking in design teams by Stempfle and Badke-Schaub (2002). In their two-process model, new solutions must be followed by analysis or evaluation, and analysis must be followed by evaluation. Such constraints within the model prevent mapping of the aforementioned cycles. Again, this might be appropriate for modelling the thinking processes in design, but it does not reflect the nature of teams' observable design operations, which have a direct effect on the state of the product being design and the state of the design process.

The probabilities of sequences of ASE design operations mapped onto the state-transition visualisation reveal that, although the analysis-synthesis-evaluation sequence does appear in both activities, the often-disputed model by Asimow (1962) does not reflect the nature of conceptual design activity when using a fine-grain observational approach. The state-transition model has revealed that the analysis-synthesis-evaluation and synthesis-analysisevaluation sequences are merely two of many appearing in team conceptual design activity.

\subsection{Limitations and further work}

Although statistically significant differences have been identified between two types of team conceptual design activities, larger sample sizes are preferable in future studies to validate the hypothesised claims and patterns. Moreover, the presented research has examined only the distribution and sequences of ASE design operations, without investigating the rationale for the transitions inside and in-between the problem and the solution space. Empirical studies focused strictly on the reasoning for particular design operations could provide a further understanding of the patterns identified during the team conceptual design activities.

An additional limitation has been recognised in the lack of describing the context of team discussions. The derived patterns are based solely on the strings of design operations codes. For instance, when capturing a sequence of solution analysis following solution synthesis, it was not examined if the two design operations involve the same design entity. However, in the future, the additional dimension of discussion context could help in identifying patterns of ASE design operations related to a single or a group of related design entities, both in the problem and the solution space. For example, IMoD by Srinivasan and Chakrabarti 2010a utilises three dimensions to link the process, the design spaces and the outcomes, thus enabling tracking of activity patterns related to individual design entities. Moreover, Linkography (Goldschmidt 2014, 2016) can also be used to mark segments of activity which are related to the evolution of a single design entity.

Similarly, the study is limited in addressing design operations solely on the team level. Hence, the protocol data does not provide information on team members which took part in the sequences of design operations. Another issue which has not been investigated is the relation between the roles of team members and their individual contribution to performing design operations. It is suggested that further studies include an additional layer to the coding process, which would provide data on who is taking a turn.

The proposed state-transition model can be used to investigate the effects of design methods, environments and team members' characteristics (background, experience, motivation, etc.) on the patterns of ASE design operations performed in the problem and the solution space. Earlier studies have shown that the methods used during team design activities (Gero et al. 2013), designers' background (Jiang et al. 2014; Gero and Jiang 2016) and the type of communication (virtual vs face-to-face) (Gero and Kan 2016) can affect the team's design process.

Further work should also inspect the applicability of the model to describe team activities in different phases of the design process. In the presented study the focus was set on conceptual design activities since the concept design phase has been regarded as critical for the parallel development of the problem and the solution space. Nonetheless, it is argued that team activities in the stages of planning, embodiment or detailed design could also be investigated using the proposed state-transition model.

In addition, the transitions of states have been explored solely by analysing the overall proportions of design operations throughout the sessions. Further steps might investigate how the proportions of design operations and the probabilities of one design operations following another one change with the progress of the design activity. Such insights could lead to hypothesising proportions and transition probabilities of design operations as functions of time or the number of design entities present within the design space.

Finally, the rationale for the probabilities of specific transitions (design operations) between the states could be hypothesised and investigated. For example, synthesis of a new design entity might be studied as a result of association, transformation or memory-based thinking processes of designers (Sauder and Jin 2016; Goldschmidt 2016). Similarly, analysis as a result of questioning and misunderstanding (Stempfle and Badke-Schaub 2002; Sauder and Jin 2016), and evaluation resulting from the need for narrowing the design space (see, e.g. McComb et al. 2015 and Yilmaz and Daly 2016) can be investigated.

\section{Conclusion}

The development and utilisation of a state-transition model revealed the proportions and the sequences of ASE design operations which are expected during team conceptual design activities. Additionally, the activity-specific sequences which differentiate ideation from concept review have been identified. Given the iterative nature of designing and the ill-defined nature of design problems in the conceptual design phase, it is unsurprising that 
neither the observed ideation or the concept review activities followed the microscale cycles of analysis-synthesisevaluation or synthesis-analysis-evaluation, as suggested by some of the reviewed models.

Analysis, synthesis and evaluation have been interpreted as fundamental mechanisms that design teams apply to evolve the content of the design space, irrespective of its problem and solution dimensions. The presented analysis revealed that design teams utilise similar sequences of ASE design operations as they progressively explore the problem and the solution space during ideation. Despite the relatively low proportion of problem-related discussion during concept review, it has been shown that design operations in problem space play an important role within the refinement and convergence cycles. Hence, the conceptualisation of ASE as design operations performed similarly in the problem and the solution space provided new insights which complement the research on the coevolution of the two spaces.

Based on the listed findings, it is argued that the developed state-transition model provides more flexibility when it comes to capturing and comparing the patterns of ASE design operations in the problem and the solution space and offers the potential of improving the understanding of the design process through either empirical or computational studies of team conceptual design activity.

Finally, the proposed visualisation of state-transitions is argued to augment the understanding of design operation patterns emerging during team conceptual design activities in two ways. First, as a summary of moves between ASE design operations within and in-between the problem and the solution space, where line thickness and colour coding are utilised to depict the frequency and types of transitions between the states of the explored design space. Second, as a template for mapping and visualising both the common and the activity-specific patterns of design operation sequences that can be identified during team conceptual designing.

Acknowledgments: This paper reports on work funded by the Croatian Science Foundation MInMED (http://www.minmed.org) and TAIDE projects (http://www.taide.org). The authors would like to thank Philip Cash (TU Denmark) for providing the multimedia data of the experiment sessions.

Funding: Croatian Science Foundation project IP-2018-01-7269: Team Adaptability for Innovation-Oriented Product Development - TAIDE (http://www.taide.org).

Conflict of Interest: The authors declare that they have no conflict of interest.

\section{Appendix}

See Tables 10 and 11.

Table 10 Averaged proportions of moves between ASE design operations in problem and solution space (left) and aggregated to ASE (right), obtained from the ideation and concept review activities

ASE design operations in problem and solution space

ASE design operations

\begin{tabular}{|c|c|c|c|c|c|c|c|}
\cline { 2 - 7 } \multicolumn{1}{c|}{} & PA & PS & PE & SA & SS & SE \\
\hline \multirow{2}{*}{} & PA & 0.018 & 0.048 & 0.026 & 0.002 & 0.038 & 0.005 \\
\cline { 2 - 7 } & PS & 0.051 & 0.047 & 0.031 & 0.003 & 0.033 & 0.005 \\
\hline PE & 0.022 & 0.019 & 0.002 & 0.003 & 0.018 & 0.001 \\
\hline SA & 0.014 & 0.021 & 0.002 & 0.020 & 0.077 & 0.046 \\
\hline SS & 0.018 & 0.021 & 0.002 & 0.122 & 0.099 & 0.056 \\
\hline \multirow{2}{*}{} & SE & 0.011 & 0.016 & 0.000 & 0.031 & 0.053 & 0.018 \\
\hline
\end{tabular}

\begin{tabular}{|c|c|c|c|}
\cline { 2 - 4 } \multicolumn{1}{c|}{} & Analysis & Synthesis & Evaluation \\
\hline Analysis & 0.054 & 0.184 & 0.079 \\
\hline Synthesis & 0.195 & 0.200 & 0.095 \\
\hline Evaluation & 0.066 & 0.106 & 0.022 \\
\hline
\end{tabular}

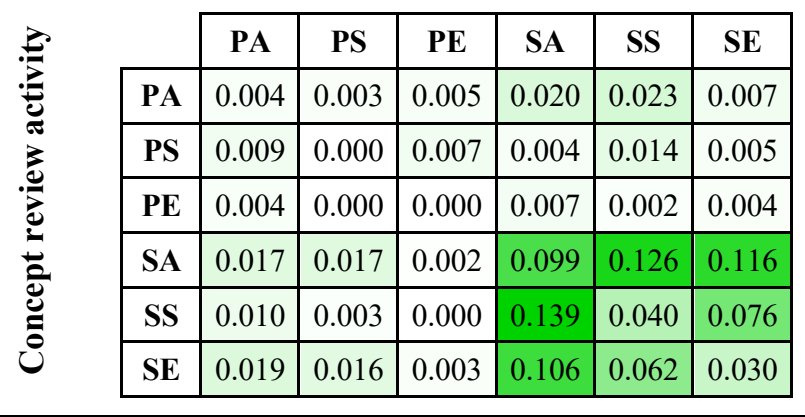

\begin{tabular}{|c|c|c|c|}
\cline { 2 - 4 } \multicolumn{1}{c|}{} & Analysis & Synthesis & Evaluation \\
\hline Analysis & 0.140 & 0.170 & 0.129 \\
\hline Synthesis & 0.162 & 0.057 & 0.088 \\
\hline Evaluation & 0.136 & 0.081 & 0.037 \\
\hline
\end{tabular}


Table 11 Averaged proportions of sequences of three consecutive design operations obtained from the ideation and concept review activities

\begin{tabular}{|c|c|c|c|c|c|c|c|c|c|c|c|c|c|}
\hline & \multicolumn{6}{|c|}{ Ideation activity } & \multicolumn{7}{|c|}{ Concept review activity } \\
\hline & PA & PS & PE & SA & SS & SE & & PA & PS & $\mathbf{P E}$ & SA & SS & SE \\
\hline $\mathbf{P A} \rightarrow \mathbf{P A}$ & 0.004 & 0.007 & 0.003 & 0.000 & 0.003 & 0.002 & $\mathbf{A} \rightarrow \mathbf{P A}$ & 0.000 & 0.001 & 0.001 & 0.002 & 0.000 & 0.000 \\
\hline $\mathbf{P A} \rightarrow \mathbf{P S}$ & 0.015 & 0.014 & 0.011 & 0.001 & 0.006 & 0.000 & $\mathrm{~A} \rightarrow \mathrm{PS}$ & 0.000 & 0.000 & 0.002 & 0.000 & 0.001 & 0.001 \\
\hline $\mathbf{P A} \rightarrow \mathbf{P E}$ & 0.008 & 0.009 & 0.000 & 0.001 & 0.008 & 0.001 & $\mathbf{P A} \rightarrow \mathbf{P E}$ & 0.002 & 0.000 & 0.000 & 0.000 & 0.002 & 0.001 \\
\hline $\mathbf{P A} \rightarrow \mathbf{S A}$ & 0.001 & 0.000 & 0.000 & 0.000 & 0.001 & 0.000 & $\mathbf{P A} \rightarrow \mathbf{S A}$ & 0.002 & 0.001 & 0.000 & 0.004 & 0.007 & 0.007 \\
\hline $\mathbf{P A} \rightarrow \mathbf{S S}$ & 0.002 & 0.000 & 0.000 & 0.012 & 0.016 & 0.009 & $\mathbf{P A} \rightarrow \mathbf{S S}$ & 0.002 & 0.000 & 0.000 & 0.010 & 0.006 & 0.005 \\
\hline $\mathbf{P A} \rightarrow \mathbf{S E}$ & 0.000 & 0.000 & 0.000 & 0.002 & 0.001 & 0.001 & $\mathbf{P A} \rightarrow \mathrm{SE}$ & 0.001 & 0.001 & 0.000 & 0.003 & 0.002 & 0.001 \\
\hline $\mathbf{P S} \rightarrow \mathbf{P A}$ & 0.006 & 0.023 & 0.009 & 0.000 & 0.013 & 0.001 & $\mathbf{P S} \rightarrow \mathbf{P A}$ & 0.001 & 0.000 & 0.001 & 0.003 & 0.004 & 0.000 \\
\hline PS $\rightarrow$ PS & 0.018 & 0.022 & 0.003 & 0.000 & 0.004 & 0.002 & $\mathrm{PS} \rightarrow \mathrm{PS}$ & 0.000 & 0.000 & 0.000 & 0.000 & 0.000 & 0.000 \\
\hline $\mathbf{P S} \rightarrow \mathbf{P E}$ & 0.011 & 0.008 & 0.003 & 0.002 & 0.009 & 0.000 & $\mathrm{PS} \rightarrow \mathrm{PE}$ & 0.002 & 0.000 & 0.000 & 0.005 & 0.000 & 0.001 \\
\hline $\mathrm{PS} \rightarrow \mathrm{SA}$ & 0.000 & 0.000 & 0.000 & 0.002 & 0.001 & 0.001 & $\mathbf{P S} \rightarrow \mathbf{S A}$ & 0.000 & 0.000 & 0.000 & 0.000 & 0.003 & 0.002 \\
\hline $\mathrm{PS} \rightarrow \mathrm{SS}$ & 0.002 & 0.003 & 0.000 & 0.012 & 0.009 & 0.008 & $\mathrm{PS} \rightarrow \mathrm{SS}$ & 0.001 & 0.001 & 0.000 & 0.007 & 0.000 & 0.005 \\
\hline $\mathrm{PS} \rightarrow \mathrm{SE}$ & 0.001 & 0.000 & 0.000 & 0.002 & 0.002 & 0.001 & $\mathrm{PS} \rightarrow \mathrm{SE}$ & 0.000 & 0.000 & 0.000 & 0.003 & 0.002 & 0.001 \\
\hline $\mathrm{PE} \rightarrow \mathrm{PA}$ & 0.003 & 0.006 & 0.006 & 0.000 & 0.006 & 0.000 & $\mathrm{PE} \rightarrow \mathrm{PA}$ & 0.000 & 0.001 & 0.001 & 0.000 & 0.002 & 0.001 \\
\hline $\mathrm{PE} \rightarrow \mathrm{PS}$ & 0.006 & 0.004 & 0.004 & 0.001 & 0.003 & 0.000 & $\mathrm{PE} \rightarrow \mathrm{PS}$ & 0.000 & 0.000 & 0.000 & 0.000 & 0.000 & 0.000 \\
\hline $\mathrm{PE} \rightarrow \mathrm{PE}$ & 0.001 & 0.001 & 0.000 & 0.000 & 0.001 & 0.000 & $\mathrm{PE} \rightarrow \mathrm{PE}$ & 0.000 & 0.000 & 0.000 & 0.000 & 0.000 & 0.000 \\
\hline $\mathbf{P E} \rightarrow \mathbf{S A}$ & 0.000 & 0.001 & 0.001 & 0.000 & 0.001 & 0.000 & $\mathbf{P E} \rightarrow \mathbf{S A}$ & 0.000 & 0.000 & 0.000 & 0.002 & 0.001 & 0.003 \\
\hline $\mathrm{PE} \rightarrow \mathrm{SS}$ & 0.002 & 0.001 & 0.000 & 0.010 & 0.005 & 0.001 & $\mathrm{PE} \rightarrow \mathrm{SS}$ & 0.000 & 0.000 & 0.000 & 0.002 & 0.000 & 0.001 \\
\hline $\mathrm{PE} \rightarrow \mathrm{SE}$ & 0.000 & 0.000 & 0.000 & 0.000 & 0.001 & 0.000 & $\mathrm{PE} \rightarrow \mathrm{SE}$ & 0.001 & 0.001 & 0.000 & 0.001 & 0.000 & 0.001 \\
\hline $\mathbf{S A} \rightarrow \mathbf{P A}$ & 0.001 & 0.005 & 0.005 & 0.000 & 0.003 & 0.000 & $\mathbf{S A} \rightarrow \mathbf{P A}$ & 0.002 & 0.002 & 0.000 & 0.006 & 0.005 & 0.003 \\
\hline $\mathrm{SA} \rightarrow \mathrm{PS}$ & 0.005 & 0.003 & 0.003 & 0.001 & 0.009 & 0.000 & $\mathbf{S A} \rightarrow \mathbf{P S}$ & 0.006 & 0.000 & 0.004 & 0.002 & 0.006 & 0.000 \\
\hline $\mathrm{SA} \rightarrow \mathrm{PE}$ & 0.001 & 0.001 & 0.000 & 0.000 & 0.000 & 0.000 & $\mathrm{SA} \rightarrow \mathrm{PE}$ & 0.001 & 0.000 & 0.000 & 0.000 & 0.000 & 0.001 \\
\hline $\mathbf{S A} \rightarrow \mathbf{S A}$ & 0.000 & 0.000 & 0.001 & 0.005 & 0.006 & 0.009 & $\mathbf{S A} \rightarrow \mathbf{S A}$ & 0.004 & 0.006 & 0.000 & 0.030 & 0.034 & 0.024 \\
\hline $\mathrm{SA} \rightarrow \mathrm{SS}$ & 0.003 & 0.007 & 0.000 & 0.028 & 0.027 & 0.012 & $\mathrm{SA} \rightarrow \mathrm{SS}$ & 0.001 & 0.001 & 0.000 & 0.068 & 0.018 & 0.039 \\
\hline $\mathrm{SA} \rightarrow \mathrm{SE}$ & 0.003 & 0.005 & 0.000 & 0.008 & 0.020 & 0.009 & $\mathrm{SA} \rightarrow \mathrm{SE}$ & 0.009 & 0.006 & 0.002 & 0.050 & 0.036 & 0.011 \\
\hline $\mathrm{SS} \rightarrow \mathrm{PA}$ & 0.003 & 0.003 & 0.002 & 0.001 & 0.010 & 0.001 & $\mathrm{SS} \rightarrow \mathrm{PA}$ & 0.000 & 0.000 & 0.000 & 0.003 & 0.004 & 0.003 \\
\hline $\mathrm{SS} \rightarrow \mathrm{PS}$ & 0.003 & 0.002 & 0.006 & 0.000 & 0.006 & 0.003 & $\mathrm{SS} \rightarrow \mathrm{PS}$ & 0.001 & 0.000 & 0.000 & 0.001 & 0.001 & 0.000 \\
\hline $\mathrm{SS} \rightarrow \mathrm{PE}$ & 0.001 & 0.001 & 0.000 & 0.000 & 0.001 & 0.000 & $\mathrm{SS} \rightarrow \mathrm{PE}$ & 0.000 & 0.000 & 0.000 & 0.000 & 0.000 & 0.000 \\
\hline $\mathbf{S S} \rightarrow$ SA & 0.012 & 0.016 & 0.000 & 0.009 & 0.059 & 0.026 & $\mathrm{SS} \rightarrow \mathrm{SA}$ & 0.008 & 0.006 & 0.000 & 0.033 & 0.043 & 0.049 \\
\hline $\mathrm{SS} \rightarrow \mathrm{SS}$ & 0.004 & 0.007 & 0.001 & 0.041 & 0.027 & 0.018 & $\mathrm{SS} \rightarrow \mathrm{SS}$ & 0.001 & 0.001 & 0.000 & 0.020 & 0.010 & 0.009 \\
\hline $\mathrm{SS} \rightarrow \mathrm{SE}$ & 0.004 & 0.009 & 0.000 & 0.012 & 0.026 & 0.004 & $\mathrm{SS} \rightarrow \mathrm{SE}$ & 0.005 & 0.005 & 0.002 & 0.038 & 0.018 & 0.007 \\
\hline $\mathrm{SE} \rightarrow \mathrm{PA}$ & 0.002 & 0.002 & 0.002 & 0.001 & 0.004 & 0.001 & $\mathrm{SE} \rightarrow \mathrm{PA}$ & 0.001 & 0.000 & 0.002 & 0.006 & 0.009 & 0.000 \\
\hline $\mathrm{SE} \rightarrow \mathrm{PS}$ & 0.004 & 0.002 & 0.004 & 0.001 & 0.005 & 0.000 & $\mathrm{SE} \rightarrow \mathrm{PS}$ & 0.002 & 0.000 & 0.002 & 0.002 & 0.007 & 0.004 \\
\hline $\mathrm{SE} \rightarrow \mathrm{PE}$ & 0.000 & 0.000 & 0.000 & 0.000 & 0.000 & 0.000 & $\mathrm{SE} \rightarrow \mathrm{PE}$ & 0.000 & 0.000 & 0.000 & 0.002 & 0.001 & 0.001 \\
\hline $\mathrm{SE} \rightarrow \mathrm{SA}$ & 0.001 & 0.004 & 0.000 & 0.004 & 0.010 & 0.011 & $\mathrm{SE} \rightarrow \mathrm{SA}$ & 0.004 & 0.004 & 0.002 & 0.030 & 0.037 & 0.030 \\
\hline $\mathrm{SE} \rightarrow \mathrm{SS}$ & 0.007 & 0.002 & 0.001 & 0.019 & 0.016 & 0.009 & $\mathrm{SE} \rightarrow \mathrm{SS}$ & 0.005 & 0.000 & 0.000 & 0.034 & 0.007 & 0.017 \\
\hline $\mathrm{SE} \rightarrow \mathrm{SE}$ & 0.003 & 0.002 & 0.000 & 0.007 & 0.003 & 0.002 & $\mathrm{SE} \rightarrow \mathrm{SE}$ & 0.003 & 0.003 & 0.000 & 0.013 & 0.004 & 0.009 \\
\hline
\end{tabular}




\section{References}

Adams RS, Cardella M, Purzer S (2016) Analyzing design review conversations: Connecting design knowing, being and coaching. Des Stu 45:1-8. https://doi.org/10.1016/j.destud.2016.03.001

Afacan Y, Demirkan H (2011) An ontology-based universal design knowledge support system. Knowledge-Based Systems 24(4):530-541. https://doi.org/10.1016/j.knosys.2011.01.002

Andreasen MM, Hansen CT, Cash P (2015) Conceptual design: Interpretations, mindset and models. Springer, Cham.

Asimow M (1962) Introduction to design. Prentice-Hall, Englewood Cliffs, N.J.

Aurisicchio M, Bracewell RH, Wallace KM (2013) Characterising the information requests of aerospace engineering designers. Res Eng Des 24(1):43-63. https://doi.org/10.1007/s00163-012-0136-y

Ball LJ, Onarheim B, Christensen BT (2010) Design requirements, epistemic uncertainty and solution development strategies in software design. Des Stud. 31(6):567-589. https://doi.org/10.1016/j.destud.2010.09.003

Bender B (2003) Task design and task analysis for empirical studies into design activity. J Eng Des. 14(4):399408. https://doi.org/10.1080/09544820310001606894

Brissaud D, Garro O, Poveda O (2003) Design process rationale capture and support by abstraction of criteria. Res Eng Des 14(3):162-172. https://doi.org/10.1007/s00163-003-0038-0

Cardoso C, Badke-Schaub P, Eris O (2016) Inflection moments in design discourse: How questions drive problem framing during idea generation. Des Stud 46:59-78. https://doi.org/10.1016/j.destud.2016.07.002

Casakin H, Badke-Schaub P (2015) Mental Models and Creativity in Engineering and Architectural Design Teams. In: Gero J, Hanna S (eds) Design Computing and Cognition '14. Springer, Cham, pp 155-171

Casakin H, Badke-Schaub P (2017) Sharedness of team mental models in the course of design-related interaction between architects and clients. Des Sci 3(e14):1-21. https://doi.org/10.1017/dsj.2017.15

Cash P, Maier A (2016) Prototyping with your hands: the many roles of gesture in the communication of design concepts. J Eng Des 27(1-3):118-145. https://doi.org/10.1080/09544828.2015.1126702

Cash P, Štorga M (2015) Multifaceted assessment of ideation: using networks to link ideation and design activity. J Eng Des 26(10-12):391-415. https://doi.org/10.1080/09544828.2015.1070813

Cash PJ, Hicks BJ, Culley SJ (2013) A comparison of designer activity using core design situations in the laboratory and practice. Des. Stud. 34(5):575-611. https://doi.org/10.1016/j.destud.2013.03.002

Chakrabarti A, Morgenstern S, Knaab H (2004) Identification and application of requirements and their impact on the design process: A protocol study. Res Eng Des 15(1):22-39. https://doi.org/10.1007/s00163-003-00335

Cross N (2001) Design cognition: results from protocol and other empirical studies of design activity. In: Newstatter W, McCracken M (eds) Design knowing and learning: cognition in design education. Elsevier, Oxford, pp 79-103

Cross N, Clayburn Cross A (1995) Observations of teamwork and social processes in design. Des Stud 16(2):143170. https://doi.org/10.1016/0142-694X(94)00007-Z

Deken F, Kleinsmann M, Aurisicchio M, et al (2012) Tapping into past design experiences: Knowledge sharing and creation during novice-expert design consultations. Res Eng Des 23(3):203-218. https://doi.org/10.1007/s00163-011-0123-8

Dinar M, Shah JJ, Cagan J et al (2015) Empirical Studies of Designer Thinking: Past, Present, and Future. J Mech Des 137(2):021101. https://doi.org/10.1115/1.4029025

Dorst K, Cross N (2001) Creativity in the design process: Co-evolution of problem-solution. Des Stud 22(5):425437. https://doi.org/10.1016/S0142-694X(01)00009-6

Dorst K, Vermaas PE (2005) John Gero's function-behaviour-structure model of designing: A critical analysis. Res Eng Des 16(1-2):17-26. https://doi.org/10.1007/s00163-005-0058-z

Eckert C, Clarkson J (2005) The reality of design. In: Clarkson J, Eckert C (eds) Design process improvement: A review of current practice. Springer, London, pp 1-29

Eckert CM, Stacey M, Wyatt D, Garthwaite P (2012) Change as little as possible: creativity in design by modification. J Eng Des 23(4):337-360. https://doi.org/10.1080/09544828.2011.639299

Eisenbart B, Gericke K, Blessing LTM (2017) Taking a look at the utilisation of function models in interdisciplinary design: insights from ten engineering companies. Res Eng Des 28(3):299-331. https://doi.org/10.1007/s00163-016-0242-3

Ensici A, Badke-Schaub P, Bayazit N, Lauche K (2013) Used and rejected decisions in design teamwork. CoDesign 9(2):113-131. https://doi.org/10.1080/15710882.2013.782411

Eris O, Martelaro N, Badke-Schaub P (2014) A comparative analysis of multimodal communication during design sketching in co-located and distributed environments. Des Stud 35(6):559-592. https://doi.org/10.1016/j.destud.2014.04.002

Fiorineschi L, Rotini F, Rissone P (2016) A new conceptual design approach for overcoming the flaws of functional decomposition and morphology. J Eng Des 27(7):438-468. https://doi.org/10.1080/09544828.2016.1160275 
Frankenberger E, Auer P (1997) Standardized observation of team-work in design. Res Eng Des 9(1):1-9. https://doi.org/10.1007/BF01607053

Gagniuc PA (2017) Markov Chains: From Theory to Implementation and Experimentation. John Wiley \& Sons, Hoboken, NJ

Gero JS (1990) Design Prototypes: A Knowledge Representation Schema for Design. AI Mag 11(4):26-36. https://doi.org/10.1609/aimag.v11i4.854

Gero JS, Jiang H (2016) Exploring the design cognition of concept design reviews using the FBS-based protocol analysis. In: Adams RS, Siddiqui JA (eds) Analyzing Design Review Conversations, Purdue University Press. https://doi.org/10.5703/1288284315931

Gero JS, Jiang H, Williams CB (2013) Design cognition differences when using unstructured, partially structured, and structured concept generation creativity techniques. Int J Des Creat Innov 1(4):196-214. https://doi.org/10.1080/21650349.2013.801760

Gero JS, Kan JWT (2016) Scientific Models from Empirical Design Research. In: Cash P, Stanković T, Štorga M (eds) Experimental Design Research. Springer, Cham, pp 253-270

Gero JS, Kannengiesser U (2014) The function-behaviour-structure ontology of design. In: Chakrabarti A, Blessing L (eds) An Anthology of Theories and Models of Design. Springer, London pp. 263-283. https://doi.org/10.1007/978-1-4471-6338-1_13

Goldschmidt G (2014) Linkography: Unfolding the Design Process. The MIT Press, Cambridge, MA

Goldschmidt G (2016) Linkographic Evidence for Concurrent Divergent and Convergent Thinking in Creative Design. Creat Res J 28(2):115-122. https://doi.org/10.1080/10400419.2016.1162497

Gonçalves M, Cardoso C, Badke-Schaub P (2014) What inspires designers? Preferences on inspirational approaches during idea generation. Des Stud 35(1):29-53. https://doi.org/10.1016/j.destud.2013.09.001

Harvey S, Kou CY (2013) Collective Engagement in Creative Tasks: The Role of Evaluation in the Creative Process in Groups. Adm Sci Q 58(3):346-386. https://doi.org/10.1177/0001839213498591

Hay L, Duffy AHB, McTeague C, et al (2017) A systematic review of protocol studies on conceptual design cognition: Design as search and exploration. Des Sci 3(e10):1-36. https://doi.org/10.1017/dsj.2017.11

Hatcher G, Ion W, Maclachlan R, Marlow M, Simpson B, Wilson N, Wodehouse A (2018) Using linkography to compare creative methods for group ideation. Des Stud 58:127-152. https://doi.org/10.1016/j.destud.2018.05.002

Howard TJ, Culley SJ, Dekoninck E (2008) Describing the creative design process by the integration of engineering design and cognitive psychology literature. Des Stud 29(2):160-180. https://doi.org/10.1016/j.destud.2008.01.001

Howard TJ, Dekoninck EA, Culley SJ (2010) The use of creative stimuli at early stages of industrial product innovation. Res Eng Des 21(4):263-274. https://doi.org/10.1007/s00163-010-0091-4

Hultén M, Artman H, House D (2018) A model to analyse students' cooperative idea generation in conceptual design. Int J Technol Des Educ 28(2):451-470. https://doi.org/10.1007/s10798-016-9384-X

Jiang H, Gero JS, Yen CC (2014) Exploring designing styles using a problem-solution division. In: Gero JS (ed) Design Computing and Cognition '12. Springer, Dordrecht, pp 79-94

Jin Y, Benami O (2010) Creative patterns and stimulation in conceptual design. Artif Intell Eng Des Anal Manuf 24(2):191-209. https://doi.org/10.1017/S0890060410000053

Jin Y, Chusilp P (2006) Study of mental iteration in different design situations. Des Stud 27(1):25-55. https://doi.org/10.1016/j.destud.2005.06.003

Kan JWT, Gero JS, Tang HH (2011) Measuring cognitive design activity changes during an industry team brainstorming session. In: Gero JS (ed) Design Computing and Cognition '10. Springer, Dordrecht, pp 621640

Kannengiesser U, Gero JS (2015) Is designing independent of domain? Comparing models of engineering, software and service design. Res Eng Des 26(3):253-275. https://doi.org/10.1007/s00163-015-0195-y

Khaidzir KAM, Lawson B (2013) The cognitive construct of design conversation. Res Eng Design 24(4):331-347. https://doi.org/10.1007/s00163-012-0147-8

Klonek FE, Quera V, Burba M, Kauffeld S (2016) Group interactions and time: Using sequential analysis to study group dynamics in project meetings. Group Dyn-Theor Res 20(3):209-222. https://doi.org/10.1037/gdn0000052

Kroll E (2013) Design theory and conceptual design: Contrasting functional decomposition and morphology with parameter analysis. Res Eng Des 24(2):165-183. https://doi.org/10.1007/s00163-012-0149-6

Kruger C, Cross N (2006) Solution driven versus problem driven design: strategies and outcomes. Des Stud 27(5):527-548. https://doi.org/10.1016/j.destud.2006.01.001

Kurakawa K (2004) A scenario-driven conceptual design information model and its formation. Res Eng Des 15(2):122-137. https://doi.org/10.1007/s00163-004-0050-z

Lawson B, Dorst K (2009) Design Expertise, 1st edn. Routledge, London

Liikkanen LA, Perttula M (2009) Exploring problem decomposition in conceptual design among novice designers. Des Stud 30(1):38-59. https://doi.org/10.1016/j.destud.2008.07.003

Liikkanen LA, Perttula M (2010) Inspiring design idea generation: insights from a memory-search perspective. J Eng Des 21(5):545-560. https://doi.org/10.1080/09544820802353297 
Liu A, Lu SC-Y (2014) Alternation of analysis and synthesis for concept generation. CIRP Ann-Manuf Technol 63(1):177-180. https://doi.org/10.1016/j.cirp.2014.03.094

Liu YC, Bligh T, Chakrabarti A (2003) Towards an "ideal" approach for concept generation. Des Stud 24(4):341355. https://doi.org/10.1016/S0142-694X(03)00003-6

López-Mesa B, Mulet E, Vidal R, Thompson G (2011) Effects of additional stimuli on idea-finding in design teams. J Eng Des 22(1):31-54. https://doi.org/10.1080/09544820902911366

Macmillan S, Steele J, Austin S, et al (2001) Development and verification of a generic framework for conceptual design. Des Stud 22(2):169-191. https://doi.org/10.1016/S0142-694X(00)00025-9

Maher ML, Poon J, Boulanger S (1996) Formalising Design Exploration as Co-evolution: A Combined Gene Approach. In: Gero JS, Sudweeks F (eds) Advances in Formal Design Methods for CAD. IFIP - The International Federation for Information Processing. Springer, Boston, MA, pp 3-30

Maher ML, Tang H (2003) Co-evolution as a computational and cognitive model of design. Res Eng Des 14(1):4764. https://doi.org/10.1007/s00163-002-0016-y

Mc Neill T, Gero JS, Warren J (1998) Understanding conceptual electronic design using protocol analysis. Res Eng Des 10(3):129-140. https://doi.org/10.1007/BF01607155

McComb C, Cagan J, Kotovsky K (2015) Lifting the Veil: Drawing Insights about Design Teams from a Cofnitively-inspired Computational Model. $\quad$ Des $\quad$ Stud 40:119-142. https://doi.org/10.1016/j.destud.2015.06.005

McDonnell J (1997) Descriptive models for interpreting design. Des Stud 18(4):457-473. https://doi.org/10.1016/S0142-694X(97)00012-4

McMahon C (2015) Design Informatics: Supporting Engineering Design Processes with Information Technology. J Indian Inst Sci 95(4):365-377.

McTeague C, Duffy A, Campbell G, et al (2017) An exploration of design synthesis. In: Maier A, Škec S, Kim H et al (eds) Proceedings of the 21st International Conference on Engineering Design (ICED 17) Vol 8: Human Behaviour in Design. The Design Society, Glasgow, pp 279-288

Nikander JB, Liikkanen LA, Laakso M (2014) The preference effect in design concept evaluation, Des Stud 35(5):473-499. https://doi.org/10.1016/j.destud.2014.02.006

Petersson AM, Lundberg J (2018) Developing an ideation method to be used in cross-functional interorganizational teams by means of action design research. Res Eng Des 29(3):433-457. https://doi.org/10.1007/s00163-018-0283-X

Quera V, Bakeman R, Gnisci A (2007) Observer agreement for event sequences: Methods and software for sequence alignment and reliability estimates. Behav Res 39(1):39-49. https://doi.org/10.3758/BF03192842

Reymen IMMJ, Hammer DK, Kroes PA, et al (2006) A domain-independent descriptive design model and its application to structured reflection on design processes. Res Eng Des 16(4):147-173. https://doi.org/10.1007/s00163-006-0011-9

Roozenburg NFM, Eekels J (1995) Product Design: Fundamentals and Methods, 2nd edn. John Wiley \& Sons, Chichester

Sarkar P, Chakrabarti A (2014) Ideas generated in conceptual design and their effects on creativity. Res Eng Des 25(3):185-201. https://doi.org/10.1007/s00163-014-0173-9

Sarkar P, Chakrabarti A (2017) A Model for the Process of Idea Generation. Des J 20(2):239-257. https://doi.org/10.1080/14606925.2017.1272244

Sauder J, Jin Y (2016) A qualitative study of collaborative stimulation in group design thinking. Des Sci 2(e4):125. https://doi.org/10.1017/dsj.2016.1

Smith RP, Tjandra P (1998) Experimental observation of iteration in engineering design. Res Eng Des 10(2):107117. https://doi.org/10.1007/BF01616691

Smithers, T (2002) Synthesis in designing. In: Gero JS (ed) Artificial Intelligence in Design '02. Springer, Dordrecht, pp 3-24. https://doi.org/10.1007/978-94-017-0795-4_1

Snider C, Dekoninck E, Culley S (2016) Beyond the concept: characterisations of later-stage creative behaviour in design. Res Eng Design 27(3):265-289. https://doi.org/10.1007/s00163-016-0218-3

Sonalkar N, Mabogunje A, Leifer L (2013) Developing a visual representation to characterize moment-to-moment concept generation in design teams. Int $J$ Des Creat Innov 1(2):93-108. https://doi.org/10.1080/21650349.2013.773117

Srinivasan V, Chakrabarti A (2010a) An Integrated Model of Designing. J Comput Inf Sci Eng 10(3):031013. https://doi.org/10.1115/1.3467011

Srinivasan V, Chakrabarti A (2010b) Investigating novelty-outcome relationships in engineering design. Artif Intell Eng Des Anal Manuf 24(2):161-178. https://doi.org/10.1017/S089006041000003X

Stempfle J, Badke-Schaub P (2002) Thinking in design teams - An analysis of team communication. Des Stud 23(5):473-496. https://doi.org/10.1016/S0142-694X(02)00004-2

Stompff G, Smulders F, Henze L (2016) Surprises are the benefits: reframing in multidisciplinary design teams. Des Stud 47:187-214. https://doi.org/10.1016/j.destud.2016.09.004

Sung E, Kelley TR (2018) Identifying design process patterns: a sequential analysis study of design thinking. Int J Technol Des Educ (in press). https://doi.org/10.1007/s10798-018-9448-1 
Toh CA, Miller SR (2016a) Creativity in design teams: the influence of personality traits and risk attitudes on creative concept selection. Res Eng Des 27(1):73-89. https://doi.org/10.1007/s00163-015-0207-y

Toh CA, Miller SR (2016b) Choosing creativity: the role of individual risk and ambiguity aversion on creative concept selection in engineering design. Res Eng Des 27(3):195-219. https://doi.org/10.1007/s00163-0150212-1

Toh CA, Miller SR (2015) How engineering teams select design concepts: A view through the lens of creativity. Des Stud 38:111-138. https://doi.org/10.1016/j.destud.2015.03.001

Toh CA, Miller SR, Okudan Kremer GE (2014) The Impact of Team-Based Product Dissection on Design Novelty. J Mech Des 136(4):041004. https://doi.org/10.1115/1.4026151

Vasconcelos LA Crilly N (2016) Inspiration and fixation: Questions, methods, findings, and challenges. Des Stud 42:1-32. https://doi.org/10.1016/j.destud.2015.11.001

Visser W (2009) Design: one, but in different forms. Des Stud 30(3):187-223. https://doi.org/10.1016/j.destud.2008.11.004

Vuletic T, Duffy A, Hay L, et al (2018) The challenges in computer supported conceptual engineering design. Comput. Ind. 95:22-37. https://doi.org/10.1016/j.compind.2017.11.003

Watts RD (1966) The elements of design. In: Gregory SA (ed) The design method. Springer, Boston, pp 85-95

Wiltschnig S, Christensen BT, Ball LJ (2013) Collaborative problem-solution co-evolution in creative design. Des Stud 34(5):515-542. https://doi.org/10.1016/j.destud.2013.01.002

Wodehouse AJ, Ion WJ (2010) Information use in conceptual design: Existing taxonomies and new approaches. Int J Des 4(3):53-65.

Wynn D, Clarkson J (2005) Models of designing. In: Clarkson J, Eckert C (eds) Design Process Improvement: A Review of Current Practice. Springer, London, pp 34-59

Wynn D, Clarkson P (2017) Process models in design and development. Res Eng Des 29(2):161-202. https://doi.org/10.1007/s00163-017-0262-7

Wynn DC, Eckert CM (2017) Perspectives on iteration in design and development. Res Eng Des 28(2):153-184. https://doi.org/10.1007/s00163-016-0226-3

Yilmaz S, Daly SR (2016) Feedback in concept development: Comparing design disciplines. Des Stud 45(A):137158. https://doi.org/10.1016/j.destud.2015.12.008 